\title{
Endothelial cell O-glycan deficiency causes blood/lymphatic misconnections and consequent fatty liver disease in mice
}

\author{
Jianxin Fu, ${ }^{1}$ Holger Gerhardt, ${ }^{2}$ J. Michael McDaniel, ${ }^{1}$ Baoyun Xia, ${ }^{3}$ Xiaowei Liu, ${ }^{1}$ \\ Lacramioara Ivanciu, ${ }^{1}$ Annelii Ny, ${ }^{4}$ Karlien Hermans, ${ }^{4}$ Robert Silasi-Mansat, ${ }^{1}$ Samuel McGee, ${ }^{1}$ \\ Emma Nye, ${ }^{2}$ Tongzhong Ju, ${ }^{3}$ Maria I. Ramirez, ${ }^{5}$ Peter Carmeliet, ${ }^{4}$ Richard D. Cummings, ${ }^{3}$ \\ Florea Lupu, ${ }^{1}$ and Lijun Xia ${ }^{1,6}$
}

\begin{abstract}
${ }^{1}$ Cardiovascular Biology Research Program, Oklahoma Medical Research Foundation, Oklahoma City, Oklahoma, USA. ${ }^{2}$ Vascular Biology Laboratory, Cancer Research UK London Research Institute, London, United Kingdom. 3Department of Biochemistry, Emory University School of Medicine, Atlanta, Georgia, USA. ${ }^{4}$ The Center for Transgene Technology and Gene Therapy, Katholieke Universiteit Leuven, Leuven, Belgium. ${ }^{5}$ Department of Medicine, Boston University School of Medicine, Boston, Massachusetts, USA. ${ }^{6}$ Department of Biochemistry and Molecular Biology and Oklahoma Center for Medical Glycobiology, University of Oklahoma Health Sciences Center, Oklahoma City, Oklahoma, USA.
\end{abstract}

\begin{abstract}
Mucin-type O-glycans (O-glycans) are highly expressed in vascular ECs. However, it is not known whether they are important for vascular development. To investigate the roles of EC O-glycans, we generated mice lacking T-synthase, a glycosyltransferase encoded by the gene C1galt1 that is critical for the biosynthesis of core 1-derived O-glycans, in ECs and hematopoietic cells (termed here EHC T-syn ${ }^{-/-}$mice). EHC T-syn ${ }^{-/-}$mice exhibited embryonic and neonatal lethality associated with disorganized and blood-filled lymphatic vessels. Bone marrow transplantation and EC C1galt1 transgene rescue demonstrated that lymphangiogenesis specifically requires EC O-glycans, and intestinal lymphatic microvessels in EHC T-syn ${ }^{-/-}$mice expressed a mosaic of blood and lymphatic EC markers. The level of O-glycoprotein podoplanin was significantly reduced in EHC T-syn ${ }^{-/-}$lymphatics, and podoplanin-deficient mice developed blood-filled lymphatics resembling EHC $\mathrm{T}_{\text {-syn }} /$ - defects. In addition, postnatal inactivation of C1galt1 caused blood/lymphatic vessel misconnections that were similar to the vascular defects in the EHC T-syn ${ }^{-/-}$mice. One consequence of eliminating T-synthase in ECs and hematopoietic cells was that the EHC T-syn ${ }^{-/-}$pups developed fatty liver disease, because of direct chylomicron deposition via misconnected portal vein and intestinal lymphatic systems. Our studies therefore demonstrate that EC O-glycans control the separation of blood and lymphatic vessels during embryonic and postnatal development, in part by regulating podoplanin expression.
\end{abstract}

\section{Introduction}

During embryonic development, blood vessels initially arise from endothelial precursors. These progenitors first develop into a primary capillary plexus, a process known as vasculogenesis $(1,2)$. The vascular plexus then expands by means of endothelial cell sprouting (angiogenesis) into a vascular network (1-3). Through recruitment of mural cells and vascular specialization, the vascular network matures into a highly organized blood vascular system comprising arteries, veins, and capillaries $(2,4)$. This multistep process is governed by distinct factors $(1,2,5)$. Among them, VEGFs and their receptors control endothelial cell sprouting $(2,6,7)$. The specialization of arteries and veins is primarily regulated by the Notch family and the orphan nuclear receptor COUP-TFII, respectively $(4,8,9)$. Later during vascular development, another important vascular system, the lymphatic system, also arises (10-12). Lymphatic endothelial cells differentiate from venous endothelial cells under control of the homeobox gene prospero-related homeobox 1 (PROX1) $(11,13)$. Thereafter, lymphatic endothelial cells form a lymphatic vascular network, for which VEGF-C is a key regulator (10).

Although derived from veins, lymphatic vessels develop into an independent system $(11,12)$. Blood and lymphatic systems remain

Nonstandard abbreviations used: Prox1, prospero-related homeobox 1. Conflict of interest: The authors have declared that no conflict of interest exists. Citation for this article: J. Clin. Invest. 118:3725-3737 (2008). doi:10.1172/JCI36077. distinct despite the fact that they are in close proximity and that both angiogenesis and lymphangiogenesis are active during development and tissue remodeling $(3,11,14)$. Therefore, there must be mechanisms that control the establishment and subsequently the independent integrity of these two systems. The hematopoietic signaling proteins Syk and SLP-76 regulate the separation between the blood and lymphatic vasculature during embryonic development (15). Beyond these two factors, however, little is known about the molecular mechanisms that regulate the blood and lymphatic vasculature as distinct systems.

The lymphatic system is essential for the transport of immune cells, interstitial fluids, and dietary lipids $(2,11)$. Dysfunction in the lymphatic system contributes to many pathological conditions such as edema and tumor development $(10,16)$, yet the clinical consequences of abnormal segregation of blood and lymphatic systems remain unknown.

The development of the blood and lymphatic systems is regulated by numerous glycoproteins $(1,2,17,18)$. Core 1 -derived mucin-type O-glycans (O-glycans), which are present in most tissues/cells, modify many membrane and secreted proteins (19-21). Our previous study of mice with a global deficiency of T-synthase (referred to herein as T-syn ${ }^{-/}$mice), a critical glycosyltransferase for biosynthesis of O-glycans encoded by the gene C1galt1, revealed an essential role of O-glycans during embryonic vascular development (22). However, whether O-glycosylation of endothelium, 
A

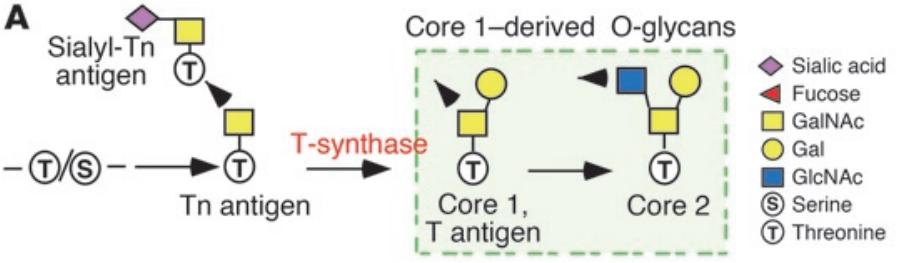

B

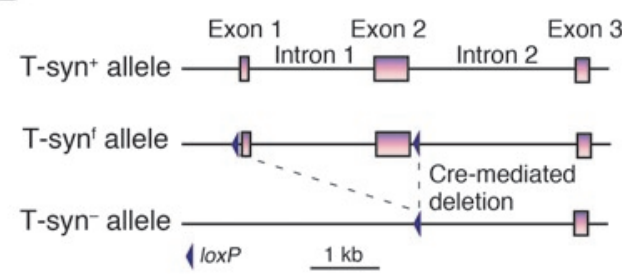

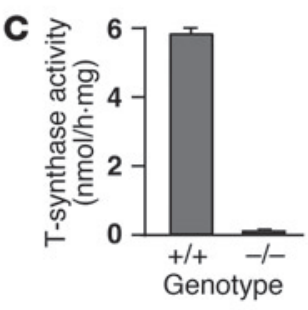

T-syn ${ }^{-1-}$

D
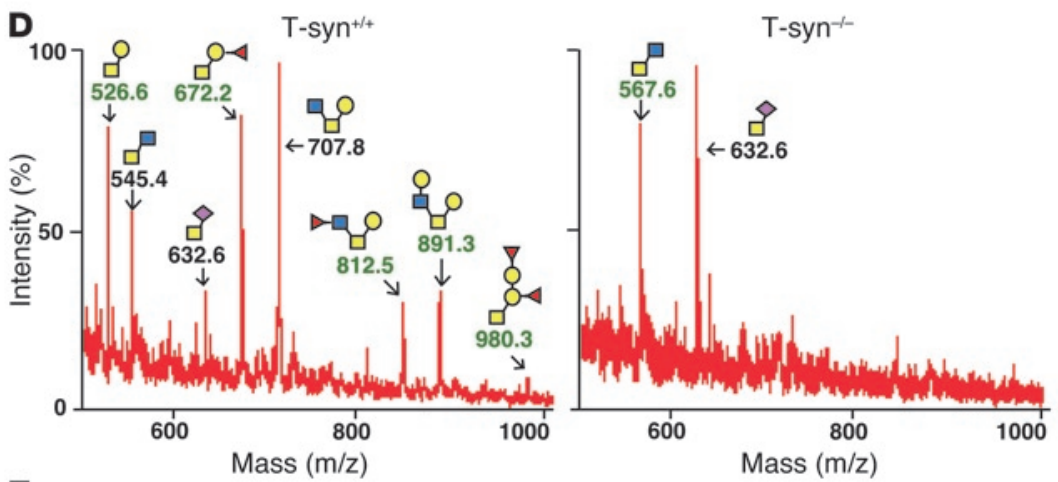

E

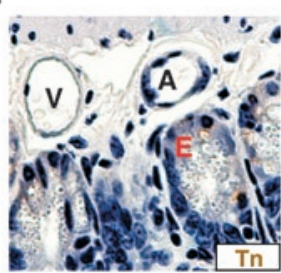

T-syn ${ }^{+/+}$

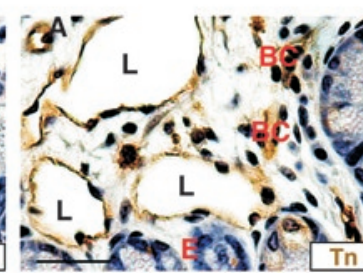

EHC T-syn ${ }^{-1-}$

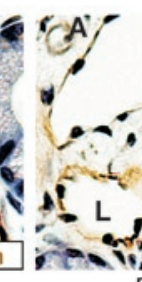

.

EHC T-syn

\section{Figure 1}

Generation of EHC T-syn ${ }^{-/-}$mice. (A) Scheme for mucin-type O-glycan biosynthesis. Arrowheads indicate possible further branching, elongation, fucosylation, sialylation, and sulfation. (B) Diagram of WT (T-syn+), loxP site-flanked (T-syn $\left.{ }^{f}\right)$, and null (T-syn $\left.{ }^{-}\right)$ alleles of C1galt1. (C) T-synthase activity of primary endothelial cells isolated from $\mathrm{T}-\mathrm{syn}^{+/+}$and $\mathrm{EHC}$

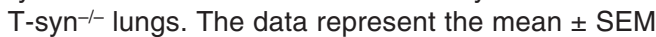
of 2 independent experiments. (D) Annotated spectra of matrix-assisted laser desorption/ionization time-offlight mass spectrometry (MALDI-TOF-MS) analyses of 2-aminobenzamide-labeled (2-AB-labeled) O-glycans from T-syn ${ }^{+/+}$and T-syn ${ }^{-/-}$endothelial cells. The O-glycans with mass number in green contain a sodium instead of a hydrogen. MALDI-TOF-MS did not detect $T n$ antigen, which is exposed in the absence of T-synthase activity, because its size is below the detection limit. (E) Immunohistochemical staining of serial intestinal sections with antibodies against Tn antigen or Lyve-1. Tn is positive in endothelial cells of EHC T-syn ${ }^{-/-}$arteriole, blood capillaries, and Lyve-1positive lymphatic vessels. $A$, arteriole; $L$, lymphatic vessels; $V$, vein; $E$, epithelium; $B C$, blood capillary. Scale bar: $50 \mu \mathrm{m}$. which expresses high levels of O-glycans, is specifically required for this process remains unknown. Moreover, the molecular targets of O-glycosylation during vascular development are not known. Here we show that mice with endothelial cell-specific deletion of C1galt1 exhibit impaired expression/function of podoplanin, an O-glycoprotein also known as T1 $\alpha$, and develop lymphatic vascular defects and abnormal lymphatic functions. This phenotype was not observed in our previous study (22), as T-syn ${ }^{-/-}$embryos die before the lymphatic system becomes functional.

\section{Results}

Mice with targeted deletion of the C1galt1 gene in endothelial and hematopoietic cells do not express endothelial O-glycans. O-glycans (Figure 1A) are highly expressed in endothelial cells (22). To investigate their contributions to vascular development, we crossed mice in which the C1galt1 gene was flanked by loxP sites (C1galt1f/f) (Figure 1B) (23) with Tie2Cre Tg mice (24). The resultant C1galt1 f/fTie2Cre ${ }^{+}$mice are deficient for T-synthase specifically in endothelial and hematopoietic cells (we refer to the mice as EHC T-syn ${ }^{-/-}$mice).

Lack of T-synthase activity in EHC T-syn ${ }^{-/}$lung primary vascular endothelial cells indicated that the Cre-mediated in vivo dele- tion was efficient (Figure 1C). Matrix-assisted laser desorption/ ionization time-of-flight mass spectrometry (MALDI-TOF-MS) revealed that $\mathrm{O}$-glycans were abundant in cultured WT $\left(\mathrm{T}-\mathrm{syn}^{+/+}\right)$ but not T-syn ${ }^{-/-}$endothelial cells (Figure 1D and Supplemental Figure 1; supplemental material available online with this article; doi:10.1172/JCI36077DS1), indicating that T-synthase is a critical glycosyltransferase for O-glycan biosynthesis in endothelial cells. To determine the specificity of the deletion, we probed EHC $\mathrm{T}^{-} \mathrm{syn}^{-/}$- intestinal tissue sections with a mAb against Tn antigen, a truncated $\mathrm{O}$-glycan structure expressed in the absence of T-synthase activity (Figure 1A). T-syn ${ }^{+/+}$tissue should not express Tn antigen. In EHC T-syn ${ }^{-/-}$mice, specific Cre-mediated deletion of C1galt1 will abolish the synthesis of O-glycans and expose Tn antigen in blood and lymphatic endothelial cells and in hematopoietic cells but not in other cell types. As expected, T-syn ${ }^{+/+}$ tissues did not express Tn antigen (Figure 1E and Supplemental Figure 1B). In EHC T-syn ${ }^{-/}$mice, Tn antigen was expressed in endothelial cells of artery, vein, lymphatics, and in hematopoietic cells, but not in other cell types, such as hepatocytes and intestinal epithelial cells (Figure 1E, Supplemental Figure 1B, and data not shown). Enzymatic desialylation of tissue sections did not 
A

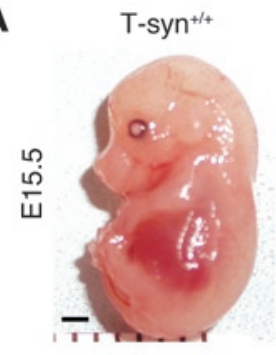

C

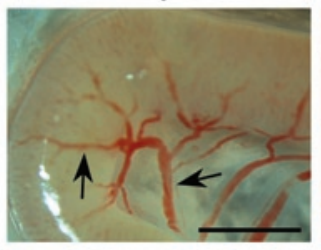

E $\quad \mathrm{T}$-syn ${ }^{+/+}$

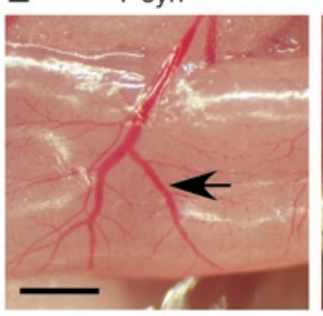

G T-syn ${ }^{+/+}$

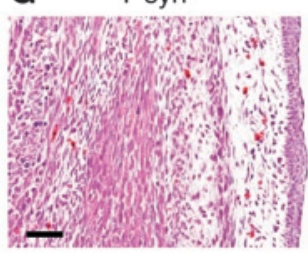

EHC T-syn ${ }^{-1}$

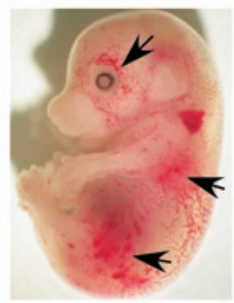

EHC T-syn ${ }^{-1-}$

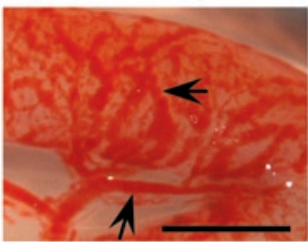

D

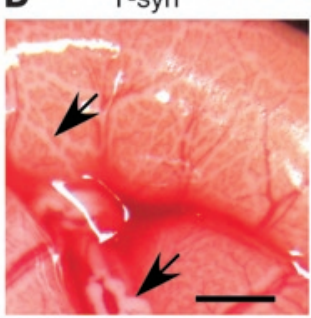

F $\quad \mathrm{T}$-syn ${ }^{+/+}$

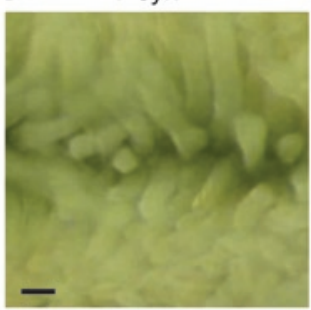

H T-syn ${ }^{+/+}$

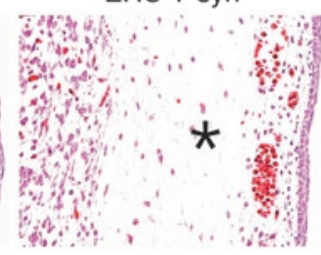

B
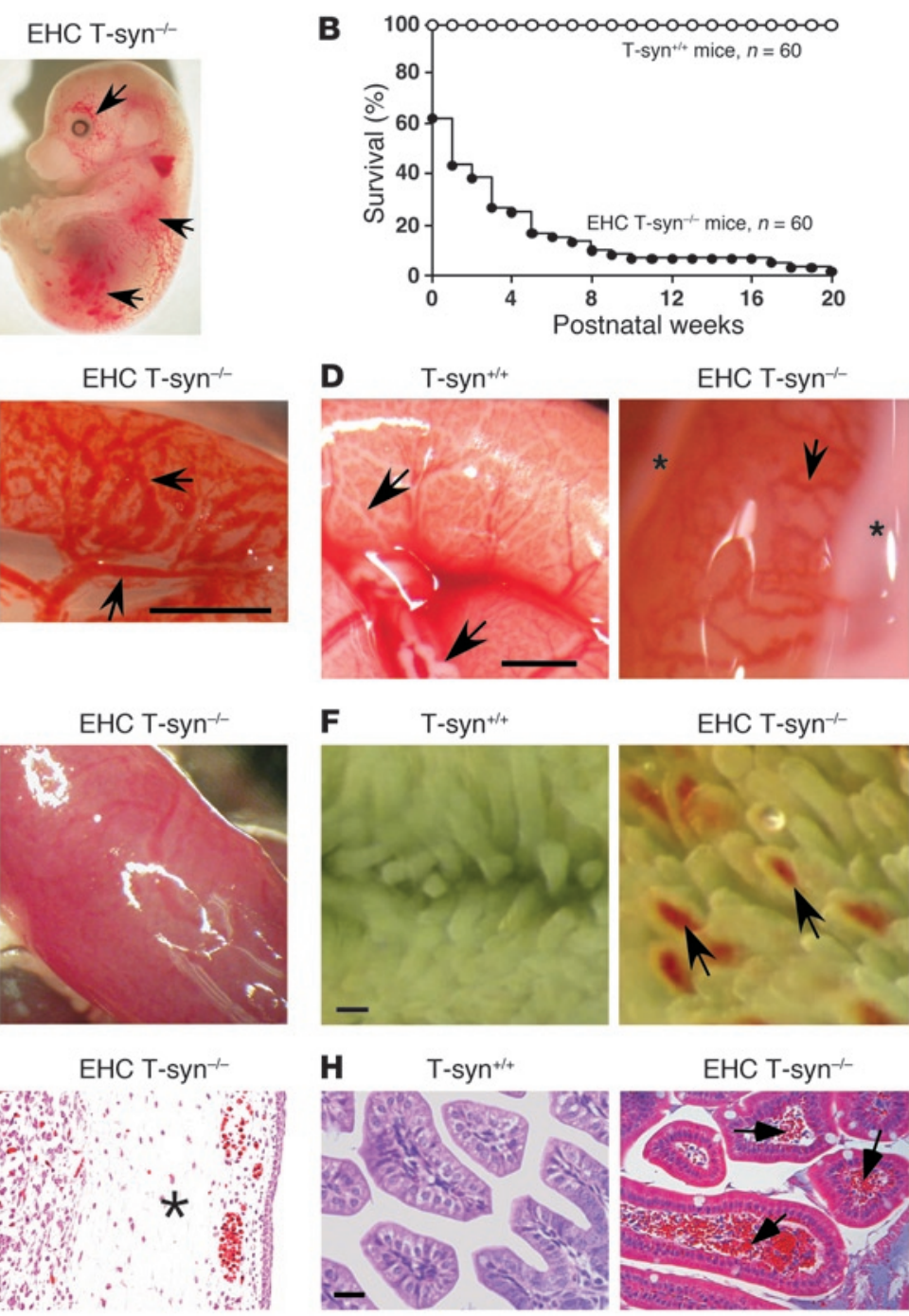

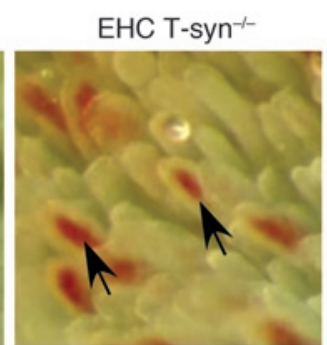

EHC T-syn ${ }^{-1-}$

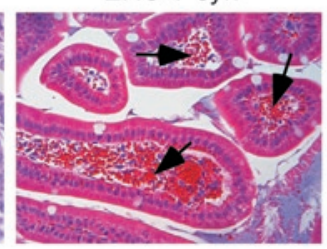

\section{Figure 2}

EHC T-syn ${ }^{-1-}$ mice exhibit high embryonic and neonatal mortality, disorganized vasculature, and impaired lymphatic function. (A) Representative E15.5 embryos showing dilated superficial vessels with scattered hemorrhages in an EHC T-syn ${ }^{-/-}$mouse compared with its T-syn ${ }^{+/+}$littermate. Arrows indicate abnormal vessels and subcutaneous bleeding. (B) Postnatal survival curves for T-syn ${ }^{+/+}$mice and EHC T-syn ${ }^{-/-}$mice. (C) Gross images of intestines of E17.5 T-syn ${ }^{+/+}$and EHC T-syn ${ }^{-/}$embryos. Arrows indicate vessels. (D) Gross images of P7 T-syn ${ }^{+/+}$and EHC T-syn ${ }^{-/-}$jeju- $^{-}$ na. Arrows indicate lymphatic vessels. Asterisks indicate chylous ascites. (E) Four-week-old T-syn ${ }^{+/+}$and EHC T-syn ${ }^{-/}$-ilea. Arrow indicates blood vessels. Widespread bleeding (overall pink color) is evident in the EHC T-syn ${ }^{-1}$ ileum. (F) Luminal surfaces of T-syn ${ }^{+/+}$and EHC T-syn ${ }^{-/-}$ilea. Arrows indicate dilated, blood-filled lacteals of EHC T-syn ${ }^{-/-}$intestinal villi. (G) Histological sections of dorsal skin of E15.5 T-syn ${ }^{+/+}$and $\mathrm{EHC}^{-\mathrm{H}}$ $\mathrm{T}$-syn $^{-1-}$ embryos. The asterisk marks enlarged subcutaneous fascia indicating edema. (H) Histological imaging of small intestine villi from 4-weekold T-syn ${ }^{+/+}$and EHC T-syn ${ }^{-/}$-mice. Arrows indicate potential blood-filled lymphatic vessels. Scale bars: $1 \mathrm{~mm}(\mathbf{A}, \mathbf{C}, \mathbf{D}$, and E); $50 \mu \mathrm{m}(\mathbf{F}-\mathbf{H})$.

expose more Tn antigen in EHC T-syn-/- tissues (data not shown), indicating little expression of the sialylated Tn antigen (Figure 1A) in EHC T-syn-/- tissues. These results indicate that the Cremediated recombination is efficient and specific in both blood and lymphatic endothelial cells (Figure 1E, right panel).

EHC T-syn ${ }^{-1-}$ mice exhibit high embryonic and neonatal mortality, disorganized vasculature, and impaired lymphatic function. EHC $\mathrm{T}^{-} \mathrm{syn}^{-/}$embryos developed normally until E14.5, after which they exhibited disorganized blood-filled vasculature with blind ends primarily in the skin and small intestine but also in other organs such as heart (Figure 2, A and C, and data not shown). Of EHC $\mathrm{T}^{-} \mathrm{syn}^{-/}$- embryos, $48 \%$ died after E14.5, and the surviving ones died during neonatal development or after weaning (Figure 2B). Of neonatal EHC T-syn ${ }^{-/-}$mice, $60 \%$ had chylous ascites (Figure 2D), but none developed bloody ascites. More than $90 \%$ of those with chylous ascites died within a week after birth. Subcutaneous edema was common in most EHC T-syn ${ }^{-/-}$embryos and surviving mice (Figure 2, A and G, and Supplemental Figure 2A). Widespread internal bleeding in small intestines was observed in approximately $75 \%$ of EHC T-syn ${ }^{-/-}$mice at autopsy (Figure $2 \mathrm{E}$ and Supplemental Figure 2B), which might be a cause of the EHC $\mathrm{T}_{-} \mathrm{syn}^{-/-}$postnatal lethality. Many EHC T-syn ${ }^{-/-}$villi had a highly dilated vascular structure (Figure $2, \mathrm{~F}$ and $\mathrm{H}$ ). Taken together, phenotypes such as chylous ascites, subcutaneous edema, and 
A

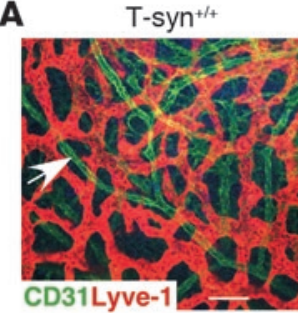

E
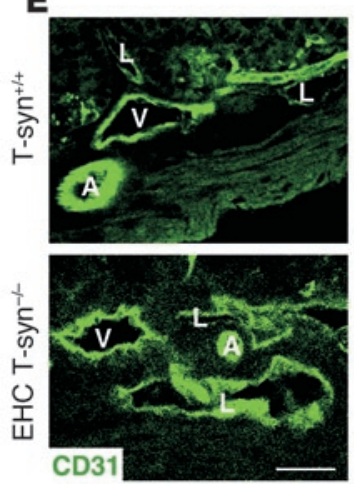

EHC T-syn ${ }^{-1}$
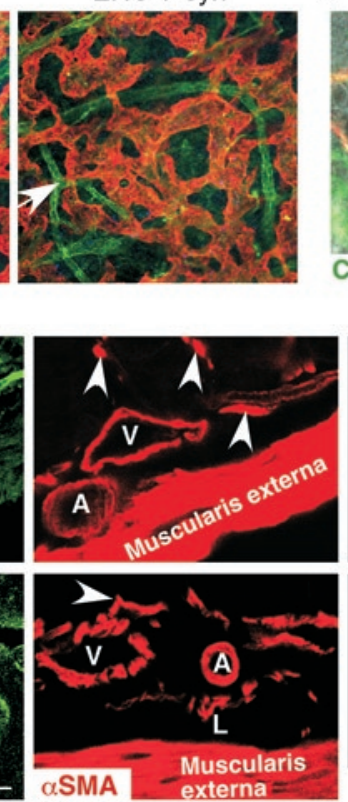

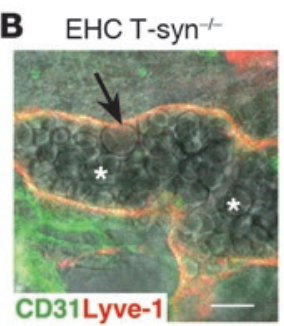

CD31Lyve-1
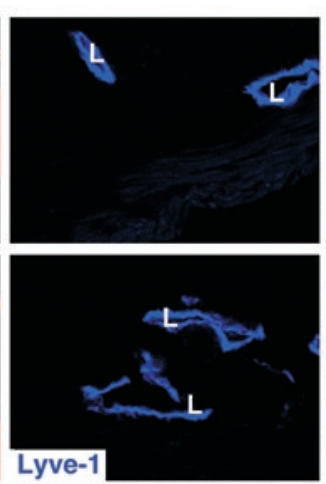
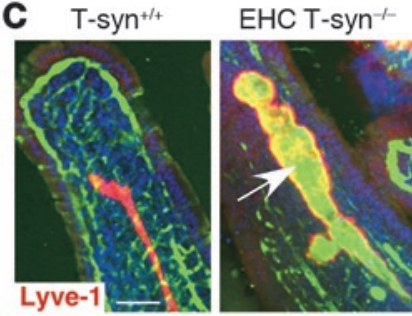

EHC T-syn-1-

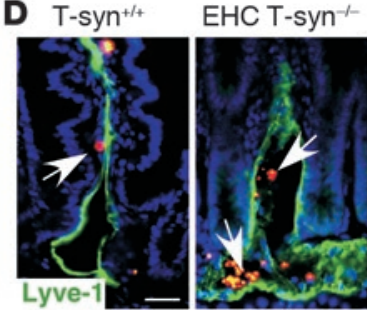

$\mathbf{F}$
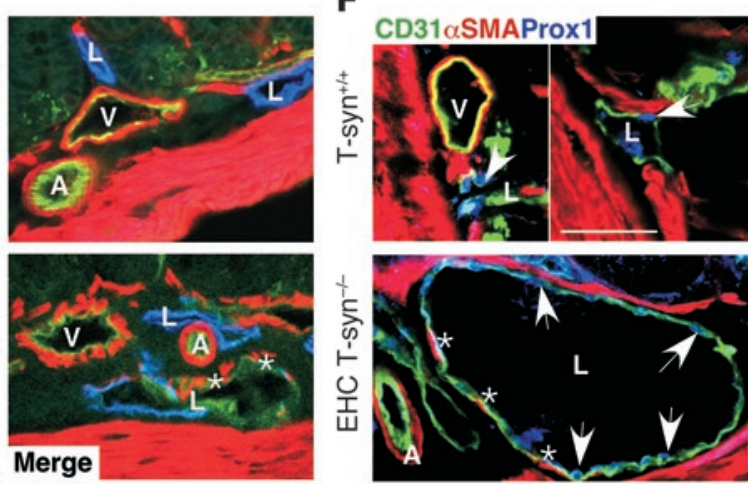

Figure 3

EHC T-syn ${ }^{-1-}$ mice have abnormal connections between blood and lymphatic vessels. (A) Whole-mount confocal imaging of E17.5 intestinal microvascular networks. A mAb against CD31 marks both blood and lymphatic vessels, and anti-Lyve-1 stains lymphatic vessels. Arrows indicate blood capillaries. (B) Overlaid confocal and phase contrast images demonstrate a blood-filled EHC T-syn ${ }^{-/-}$lymphatic vessel. Asterisks mark blood cells. Arrow marks lymphatic endothelium. (C) Microvascular networks of intestinal villi stained with anti-Lyve-1. The mice were arterially injected with dextran-FITC $(150 \mathrm{kDa})$ prior to the confocal analysis. The arrow indicates a dextran-FITC-filled EHC T-syn ${ }^{-/}$lacteal. (D) Confocal imaging of lacteals. The mice were injected with $1-\mu \mathrm{m}$ fluorescent beads (red) prior to the analysis. Arrows indicate beads. (E) Confocal images of intestinal cryosections with antibodies against CD31, $\alpha$-SMA, and Lyve-1. Arrowheads indicate smooth muscle cells in lamina propria. Asterisks indicate ectopic smooth muscle cell staining. (F) Confocal images of intestinal cryosections with antibodies against CD31, $\alpha$-SMA, and Prox1. Asterisks indicate ectopic smooth muscle cell staining. Arrows mark Prox1 staining. Scale bars: $20 \mu \mathrm{m}$. Mice were 3-6 weeks old unless otherwise specified.

abnormal blind-ended vessels in EHC T-syn ${ }^{-/-}$mice are suggestive of defective lymphatic vessel development/function.

EHC T-syn-/- mice form abnormal connections between blood and lymphatic vessels. To characterize the EHC T- $\mathrm{syn}^{-/-}$vascular abnormalities, whole-mount small intestines were stained with antibodies against CD31, a pan-endothelial marker (25), and Lyve-1, a lymphatic endothelial marker $(11,26)$. Starting from E15.5, EHC $\mathrm{T}$-syn ${ }^{-/}$mesenteric and intestinal submucosal lymphatic capillaries were disorganized compared with those of T-syn ${ }^{+/+}$mice (Figure 3A and Supplemental Figure 3A). Intriguingly, EHC T-syn-/-lymphatic vessels were filled with blood (Figure $3 \mathrm{~B}$ ), indicating potential defects in the separation of blood and lymphatic vessels. Indeed, dextran-FITC $(150 \mathrm{kDa})$ and fluorescent microbeads $(1 \mu \mathrm{m})$ injected through the carotid artery went immediately into lymphatic vessels, such as intestinal lacteals, of EHC T-syn ${ }^{-/-}$mice but not $\mathrm{T}$-syn ${ }^{+/+}$ mice (Figure 3, C and D). This confirms functional misconnections of blood and lymphatic vessels in EHC T-syn ${ }^{-1-}$ mice. There was no visible extravascular dextran-FITC in EHC T-syn ${ }^{-/-}$mice, indicating that $\mathrm{EHC} \mathrm{T-syn}{ }^{-/}$vessels were not leaky.

Analyses of cryosections of small intestines demonstrated that T-syn ${ }^{+/+}$blood endothelium expressed a high level of CD31 and no Lyve- 1 and was surrounded by $\alpha$-SMA-positive cells. T-syn ${ }^{+/+}$ intestinal submucosal lymphatic microvascular endothelium did express Lyve-1, displayed a lower level of CD31, and had no $\alpha$-SMA coating (Figure $3 \mathrm{E}$ ). The high CD31 level profile appeared unaf- fected in $\mathrm{EHC} \mathrm{T}^{-\mathrm{syn}^{-/}}$arteries, veins, and blood capillaries (Figure 3 , A and E). In contrast, most EHC T-syn ${ }^{-/-}$intestinal submucosal lymphatic capillaries were disorganized, dilated, and expressed abnormally high levels of CD31 (Figure 3E, Figure 4, A and B, and Supplemental Figure 3, A and B). All EHC T-syn-/- lymphatic capillaries expressed Prox1 (Figure 3F, Figure 4, D and E, and Supplemental Figure 3, C and D), a definitive lymphatic endothelial marker (11), yet approximately $65 \%$ of these vessels partially lost their expression of Lyve-1 (probed with an O-glycosylation-independent polyclonal antibody) (Figure 3E, Figure 4, A, D, and E, and Supplemental Figure 3, A and B). In contrast, Lyve-1 was expressed uniformly at a high level in $\mathrm{T}-\mathrm{syn}^{+/+}$intestinal lymphatic microvascular endothelium (Figure 3, A and C-E; Figure 4A; and Supplemental Figure 3, A and B). Prox1-positive lymphatic microvessels in EHC T-syn-/- intestines had ectopic staining of $\alpha$-SMA (Figure $3, \mathrm{E}$ and $\mathrm{F}$ ), which is known not to be associated with intestinal lymphatic microvessels (27). Antibodies against CD105 and endomucin, which are blood vascular endothelial cell-specific markers $(25,28,29)$, labeled only blood vascular endothelium in T-syn ${ }^{+/+}$ mice (Figure 4, E and F, and Supplemental Figure 3D). In contrast, anti-CD105 and anti-endomucin stained both EHC T-syn-/- blood and lymphatic endothelial cells, although the staining of antiendomucin was patchy (Figure 4, E and F, and Supplemental Figure 3D). Thus, the observed functional misconnections correlated with hybrid blood and lymphatic vessel marker expression within 

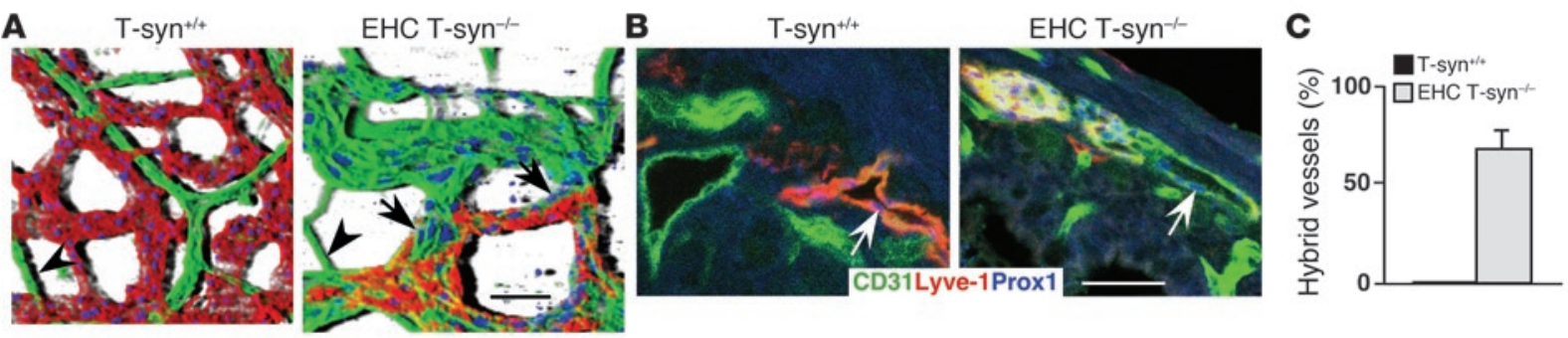

D

T-syn ${ }^{+/+}$

EHC T-syn ${ }^{-1-}$
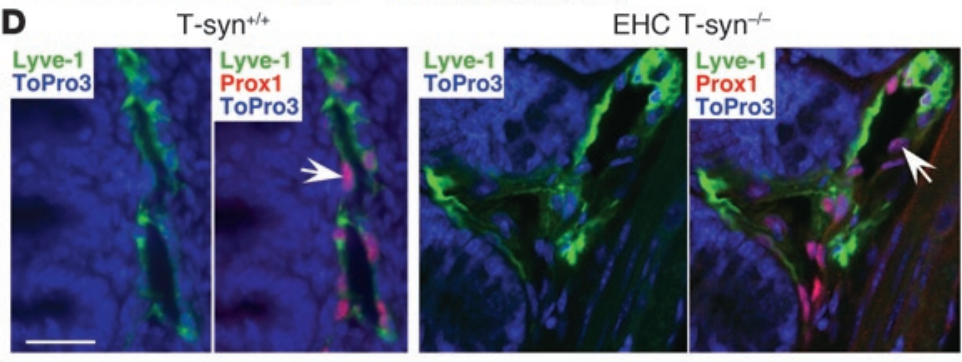

E T-syn ${ }^{+/+}$

EHC T-syn ${ }^{-1-}$

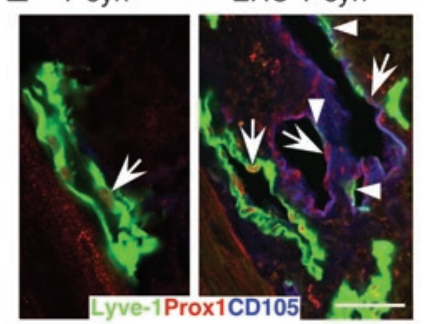

$\mathbf{F}$

T-syn $+/+$
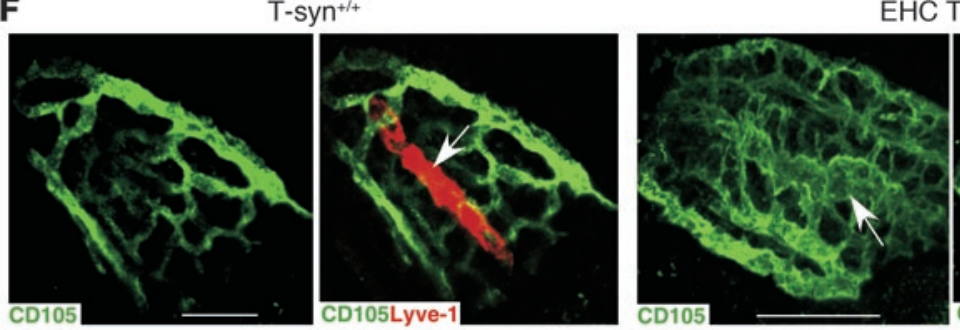

EHC T-syn ${ }^{-1-}$

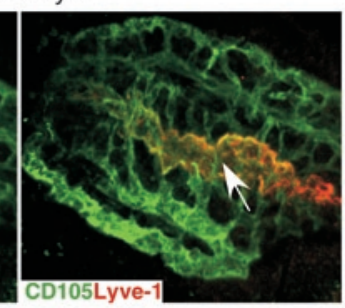

Figure 4

EHC T-syn ${ }^{-1-}$ mice develop hybrid vessels that have a mosaic expression of both blood and lymphatic endothelial cell markers. (A and B) Confocal imaging of whole-mount and cryosections of intestinal vessels. Ilea and cryosections of T-syn ${ }^{+/+}$and EHC T-syn ${ }^{-/}$mice were stained with antibodies against CD31, Lyve-1, and Prox1. Black arrows in A indicate abnormal EHC T-syn ${ }^{-1-}$ lymphatic vessels that express Prox1, some Lyve-1, and a higher level of CD31. White arrows in B mark Prox1 staining. Arrowheads indicate blood capillary. (C) Quantification of hybrid vessels in T-syn ${ }^{+/}$and EHC T-syn ${ }^{-/}$small intestines. Data are mean \pm SEM; $n=37$ images/3-5 mice/group. (D and E) Cryosections of intestinal lymphatic submucosal microvessels labeled with antibodies against Lyve-1 and Prox1 (D) or Lyve1, Prox1, and CD105 (E). ToPro3 was used for nuclear staining. Arrows indicate Prox1 staining. Arrowheads mark residual Lyve-1 staining. (F) Microvascular network and lacteal in intestinal villi labeled with anti-CD105 and anti-Lyve-1. Arrows indicate lacteals. Scale bars: $50 \mu \mathrm{m}$. Mice were 3-8 weeks old.

individual vessel profiles. The number of EHC T-syn ${ }^{-1-}$ Prox1-positive lymphatic endothelial cells appeared to be comparable with that of T-syn ${ }^{+/+}$lymphatic endothelial cells, although some of the lymphatic endothelial cells were attenuated with cytoplasmic thinning (Figure 3F, Figure 4, D and E, and Supplemental Figure 3B). These experiments indicate that endothelial O-glycans perform an important function in the segregation of blood and lymphatic endothelial cells during embryonic development.

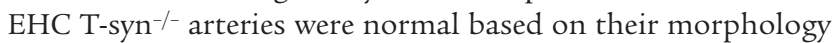
(Figure 3E). The smooth muscle cell coverage of venous endothelial cells appeared not to be as uniform in EHC T-syn-/- mice as in control T-syn ${ }^{+/+}$mice (Figure 3E). Although skin and brain blood capillaries were comparable in $\mathrm{T}-\mathrm{syn}^{+/+}$and $\mathrm{EHC} \mathrm{T-syn}{ }^{-/-}$mice (Supplemental Figure 3C and Supplemental Figure 4), EHC T-syn ${ }^{-/}$ intestinal blood capillaries had a moderate degree of abnormal branching (Figure 4F and Supplemental Figure 3A). This phenotype is similar, albeit much less severe, to the abnormal angiogenesis in global T-syn ${ }^{-/}$mice, suggesting that endothelial O-glycans contribute to angiogenesis.

Endothelial O-glycans are specifically required for vascular development. To differentiate the contribution of endothelial cells and hematopoietic cells to abnormal vascular development in EHC
T-syn ${ }^{-/-}$mice, we transplanted EHC T-syn-/- bone marrow cells into lethally irradiated WT B6.SJL recipients and vice versa to generate hematopoietic cell-specific T synthase-deficient mice (HC $\left.\mathrm{T}_{-} \mathrm{syn}^{-/-}\right)$and endothelial cell-specific $\mathrm{T}$ synthase-deficient mice $\left(\right.$ EC T-syn ${ }^{-/}$) (Supplemental Figure 5A). Nine months later, HC $\mathrm{T}^{-} \mathrm{syn}^{-/}$mice did not develop vascular abnormalities, whereas the EC T-syn ${ }^{-/}$mice retained abnormal vasculature (Supplemental Figure $5 \mathrm{~B}$ ). These data indicate that WT bone marrow is unable to restore the EHC T-syn ${ }^{-/}$vascular defects and that loss of T-synthase in bone marrow cells of WT adult mice cannot induce a postnatal abnormal vascular phenotype.

We reasoned that if the absence of endothelial O-glycans causes the phenotypes in EHC T-syn ${ }^{-/-}$mice, endothelial cell-specific expression of T-synthase would rescue EHC T-syn ${ }^{-/}$embryos and correct EHC T-syn-/- lymphatic defects. To test this hypothesis, we generated $\mathrm{Tg}$ lines that expressed $\mathrm{T}$-synthase specifically in endothelial cells under control of the Tie2 promoter and enhancer (Tie2-T-syn Tg; Figure 5A) (30), which drives the expression of the transgene in all endothelial cells, only transiently in hematopoietic stem cells, but not in differentiated hematopoietic cells $(30,31)$. Crosses between two independent Tie2-T-syn Tg founders and EHC T-syn ${ }^{+/-}$mice produced rescued EHC T-syn ${ }^{-/-}$viable pups 

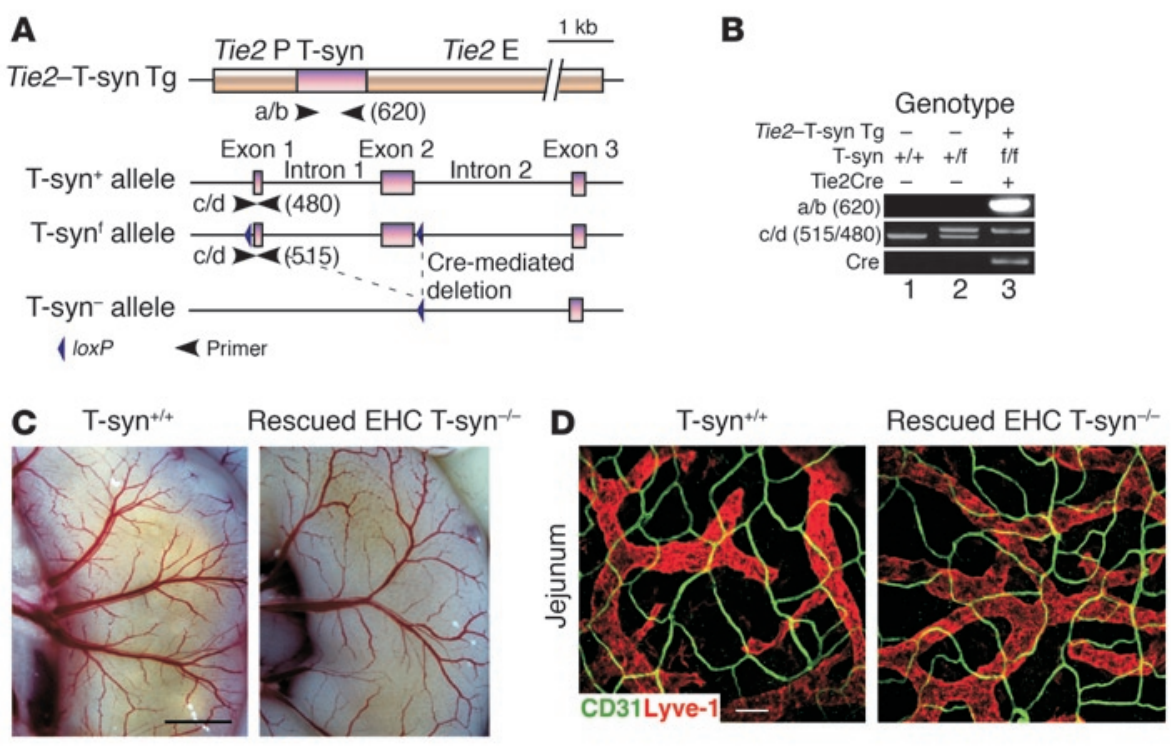

E

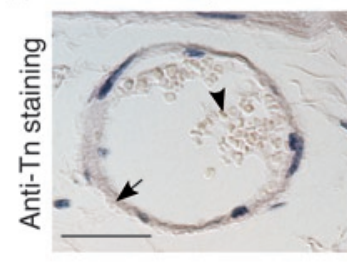

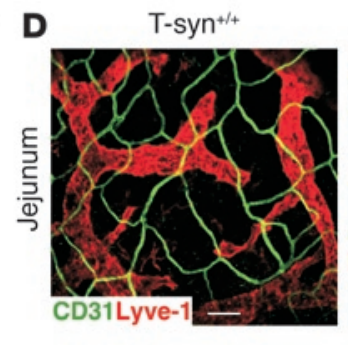

B

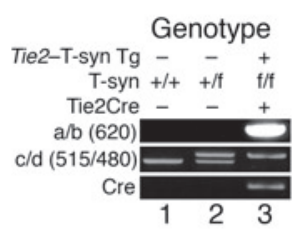

Rescued EHC T-syn ${ }^{--}$
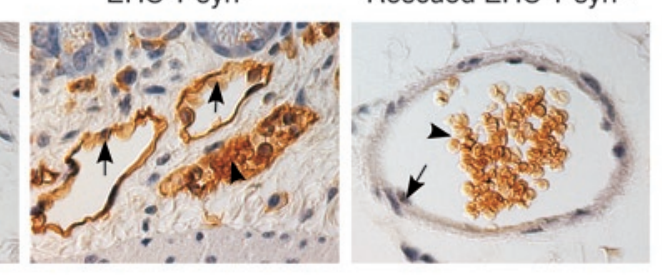

Figure 5

Endothelial expression of C1galt1 rescues EHC T-syn ${ }^{-1-}$ embryonic lethality and defective vascular development. (A) Diagram of the DNA construct used to generate Tie2-T-syn Tg mice and of T-syn+, T-syn ${ }^{f}$, and T-syn- alleles, with positions of primers used in genotyping. Tie2 $\mathrm{P}$, Tie2 promoter; Tie2 E, Tie2 intronic enhancer. (B) PCR genotyping of tail genomic DNA from offspring of Tie2-T-syn Tg crossed with $\mathrm{EHC}$ T-syn ${ }^{+/-}$mice. Lane 3 represents the genotype of the rescued EHC $\mathrm{T}^{-} \mathrm{syn}^{-/-}$mice. $\mathrm{a} / \mathrm{b}$ and $\mathrm{c} / \mathrm{d}$ indicate primers for PCR. (C) Intestinal vasculature of

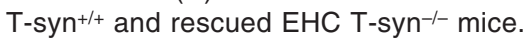
(D) Confocal imaging demonstrates that Tie2-T-syn $\mathrm{Tg}$ rescues defective lymphangiogenesis in EHC T-syn ${ }^{-/}$mice. (E) Immunohistochemical staining of intestine sections from rescued EHC T-syn ${ }^{-/}$mice with a mAb against Tn antigen. Dark brown indicates positive staining. Arrows, vascular endothelial cells; arrowheads, blood cells. Data are representative of at least 3 experiments. Scale bars: $1 \mathrm{~mm}$ (C); $50 \mu \mathrm{m}$ (D and E). Mice were 3-6 weeks old. with a normal Mendelian distribution (Figure 5B). The rescued EHC T-syn ${ }^{-/-}$mice developed normally and had normal vasculature (Figure 5, C and D). As expected, the Tn mAb did not stain the rescued $\mathrm{EHC} \mathrm{T-s^{-1 }}{ }^{--}$vascular endothelial cells but stained hematopoietic cells, whereas the mAb stained arterial, venous, and lymphatic endothelial cells and hematopoietic cells in nonrescued EHC T-syn ${ }^{-/-}$mice (Figure 5E and data not shown). This result confirms that the rescue is blood and lymphatic endothelial specific. Taken together, these experiments demonstrated that endothelial, but not hematopoietic, O-glycans are specifically required for lymphangiogenesis.

Silencing of C1galt1 in zebrafish and tadpole reveals existence of an additional C1galt1 gene. To verify the lymphatic abnormalities revealed by EHC T-syn ${ }^{-/-}$mice in different organisms, we silenced the known C1galt1 gene in zebrafish (Zebra danio) and tadpole (Xenopuslaevis). The C1galt1 knockdown was efficient in both organisms (Supplemental Figure 6A and Supplemental Figure 7, A and B). However, the knockdown tissues were not positive for Tn, which should be exposed in the absence of T-synthase; instead, they expressed a similar level of core $1 \mathrm{O}$-glycans as control tissues (data not shown). Moreover, comprehensive analyses revealed no significant blood and lymphatic vascular abnormalities in these C1galt1silenced organisms (Supplemental Figure 6 and 7). We found a new C1galt1 (Q08BL) in the June 2008 Zebra danio database (http:// www.ncbi.nlm.nih.gov/entrez/viewer.fcgi?val=NM_001077374) and a new EST that shares a high degree of homology with C1galt1 in Xenopus laevis (http://www.ncbi.nlm.nih.gov/entrez/viewer. fcgi? $d b=$ nucest $\& i d=88884333$; February 2006). The new Xenopus C1galt1 EST is not a "B" copy of the Xenopus laevis pseudotetraploid genome, because it is quite divergent. These results suggest that the lack of vascular phenotype after silencing of C1galt1 in both zebrafish and tadpole is due to the existence of another gene for T-synthase. Although only one functional C1galt1 gene has been identified in vertebrate and invertebrate animals (19), these findings suggest that there is a functionally redundant C1galt1 in these small aquatic vertebrate animal models.

Lack of O-glycans results in impaired expression of podoplanin, and mice without podoplanin phenocopy EHC T-syn-/- mice. Lack of O-glycans may alter the expression and/or function of many glycoproteins that regulate lymphangiogenesis. To identify the molecular targets, we first screened molecules that are important in angiogenesis and lymphangiogenesis by RT-PCR and Western blotting of primary endothelial cells or tissue lysates from $\mathrm{T}$-syn ${ }^{+/+}$and

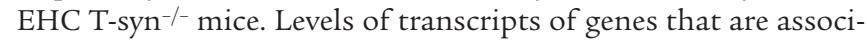
ated with vascular development were similar in T-syn ${ }^{+/+}$and $\mathrm{EHC}$ $\mathrm{T}_{-} \mathrm{syn}^{-/-}$mice (Supplemental 8A and data not shown), suggesting that abnormal O-glycosylation does not significantly affect gene expression. Among those candidate molecules, expression of podoplanin protein, a mucin-type O-glycoprotein (32), was substantially decreased in EHC T-syn ${ }^{-/}$- endothelial cells as measured by different glycosylation-independent antibodies (Figure 6A and Supplemental Figure 8B). EHC T-syn ${ }^{-/-}$endothelial podoplanin exhibited several smaller bands on a reducing SDS-PAGE gel (Figure 6A). Immunoblotting of immunoprecipitated podoplanin from primary endothelial cell and intestinal tissue lysates with the lectin Helix pomatia agglutinin (HPA), which recognizes Tn antigen, indicated that the smaller podoplanin bands were Tn positive (Figure 6, B and C), suggesting that they were underglycosylated and possibly degradation products of podoplanin. Podoplanin was markedly reduced in Lyve-1-positive intestinal submucosal 
A
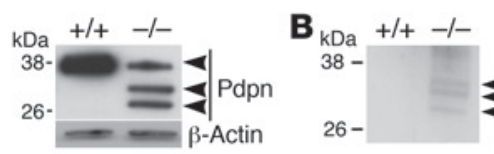

HPA-positive

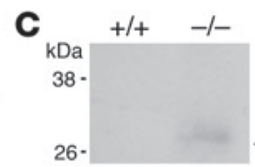

4 HPA-positive
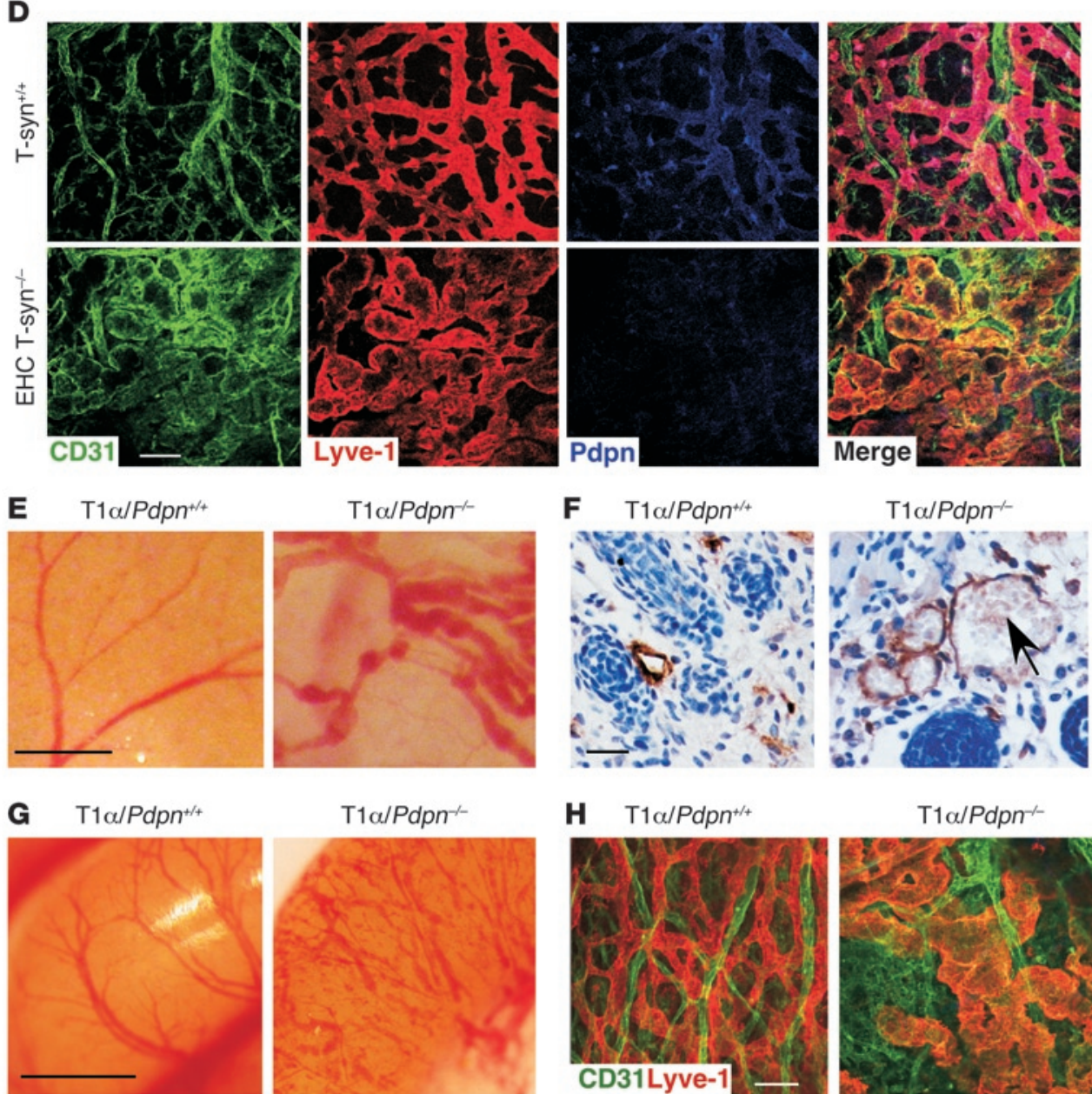

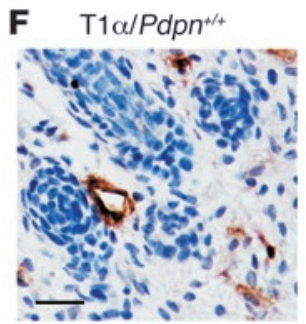

\section{H T1 $\alpha / P d p n^{+/+}$}

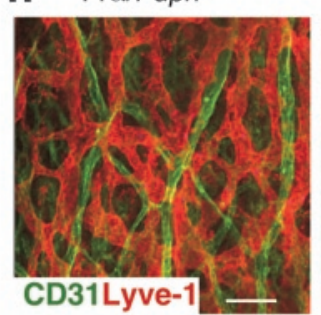

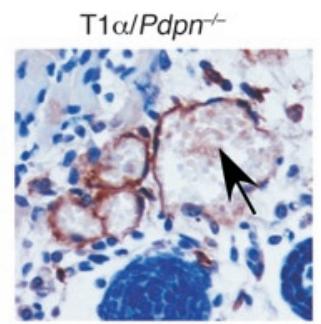

$\mathrm{T} 1 \alpha / P d p n^{--}$

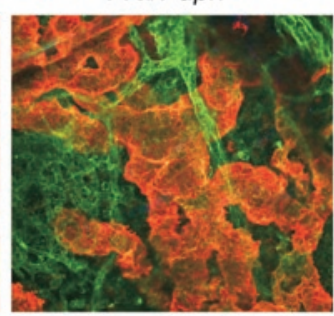

\section{Figure 6}

Impaired expression of lymphatic endothelial podoplanin (Pdpn) leads to abnormal lymphatic vascular development in EHC $\mathrm{T}^{-}$syn $^{-/-}$mice. (A) Expression of Pdpn in primary endothelial cells from T-syn ${ }^{+/+}$and EHC T-syn ${ }^{-/}$ mice was analyzed by Western blotting. $\beta$-Actin was used as a loading control. (B and C) Pdpn immunoprecipitated from primary endothelial cell lysates (B) or intestinal tissue lysates (C) and probed by Western blotting with Helix pomatia agglutinin (HPA), which detects Tn antigen. (D) Confocal analysis of Pdpn expression of intestinal lymphatic vessels of P2 mice. (E) Skin vasculature of PO WT $\left(\mathrm{Pdpn}^{+/+}\right)$and $\mathrm{Pdpn^{-/ }}$ mice. (F) Immunohistochemical staining of $\mathrm{PO} \mathrm{Pdpn} n^{+/}$and $\mathrm{Pdpn}^{-/}$ skin sections with an antibody against Lyve-1 (brown). The arrow shows blood cells in $\mathrm{Pdpn}^{-/-}$lymphatic vessels. (G) Gross vascular networks of PO Pdpn+/+ and $P d p n^{-/}$ilea. (H) Confocal imaging of lymphatic microvascular networks of ilea. Scale bars: $50 \mu \mathrm{m}$ (D, F, and $\mathbf{H}) ; 1 \mathrm{~mm}(\mathbf{E}$ and $\mathbf{G})$. Data represent results of at least 3 experiments. lymphatic endothelium in EHC T-syn ${ }^{-/-}$mice (Figure 6D). The expression of surface podoplanin was reduced in permeabilized Prox1-positive primary EHC T-syn ${ }^{-1}$ skin endothelial cells, and there was no intracellular accumulation of underglycosylated podoplanin (Supplemental Figure 9). These data suggest that O-glycosylation of endothelial cells is essential for the cell surface expression of podoplanin.

The size of Lyve-1 was smaller in EHC T-syn-/- endothelial cells than in $\mathrm{T}-\mathrm{syn}^{+/+}$endothelial cells, indicating that Lyve- 1 is O-glycosylated (Supplemental Figure 8B). Anti-Lyve-1 consistently stained Lyve- 1 in $\mathrm{T}-\mathrm{syn}^{+/+}$and EHC T-syn ${ }^{-/-}$primary endothelial cell lysates (Supplemental Figure 8B), suggesting that changes in Lyve-1 O-glycosylation do not affect its stability and that the anti-Lyve-1 epitope is not dependent on O-glycosylation.

$P d p n^{\text {tm1Mcws-/- }}$ mice $\left(P d p n^{-/}\right.$, also known as $\left.\mathrm{T}^{1 \mathrm{a}^{-/-}}\right)$die shortly after birth due to respiratory failure $(32,33)$. Although $P d p n^{-1-}$ mice have abnormal lymphatic vessels, they were not reported to have misconnections between blood and lymphatic vessels (32). We found that, $P d p n^{-/-}$mice like EHC T-syn-/- mice, had disorganized and blood-filled lymphatic vessels (Figure 6, E-H). These data sug- gest that O-glycosylated podoplanin is required for the separation of blood and lymphatic vessels during embryonic development.

Endothelial O-glycans are required for the maintenance of distinct blood and lymphatic vascular systems during postnatal development. To examine whether O-glycans are important for the continued segregation of distinct blood and lymphatic systems, we generated mice with postnatal deletion of C1galt1 (inducible T-syn ${ }^{-/}$ mice; Figure 7A). Two weeks after induction, inducible T-syn ${ }^{-1-}$ endothelial and hematopoietic cells, which normally express high levels of O-glycans, started to be positive for anti-Tn mAb staining (Figure 7B and data not shown), indicating induced deletion of C1galt1. Six months later, inducible T-syn ${ }^{-/-}$mice developed misconnections between blood and lymphatic vessels that resembled the phenotype of EHC T-syn ${ }^{-/-}$mice (Figure 7B). Intravital microscopy revealed that arterially injected India ink flowed directly from intestinal veins into lymphatic vessels, demonstrating direct connections between T-syn ${ }^{-/-}$venous and lymphatic vessels (Figure 7C and Supplemental Videos 1 and 2). As in EHC T-syn ${ }^{-/}$mice, some of the inducible $\mathrm{T}_{-} \mathrm{syn}^{-/-}$intestinal lymphatic vessels expressed a mosaic of blood and lymphatic markers (Figure 7D and data not 
A

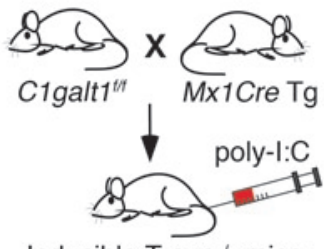

Inducible T-syn ${ }^{-1}$ mice
C

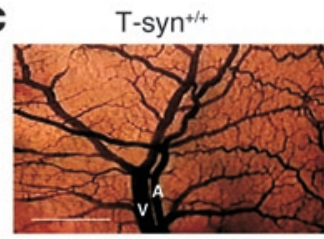

Inducible T-syn ${ }^{-1-}$

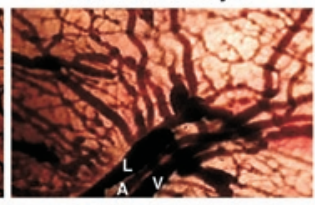

B
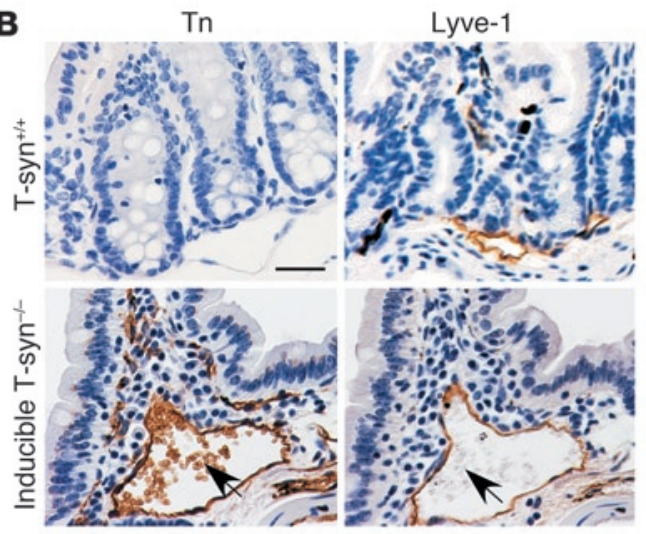

D
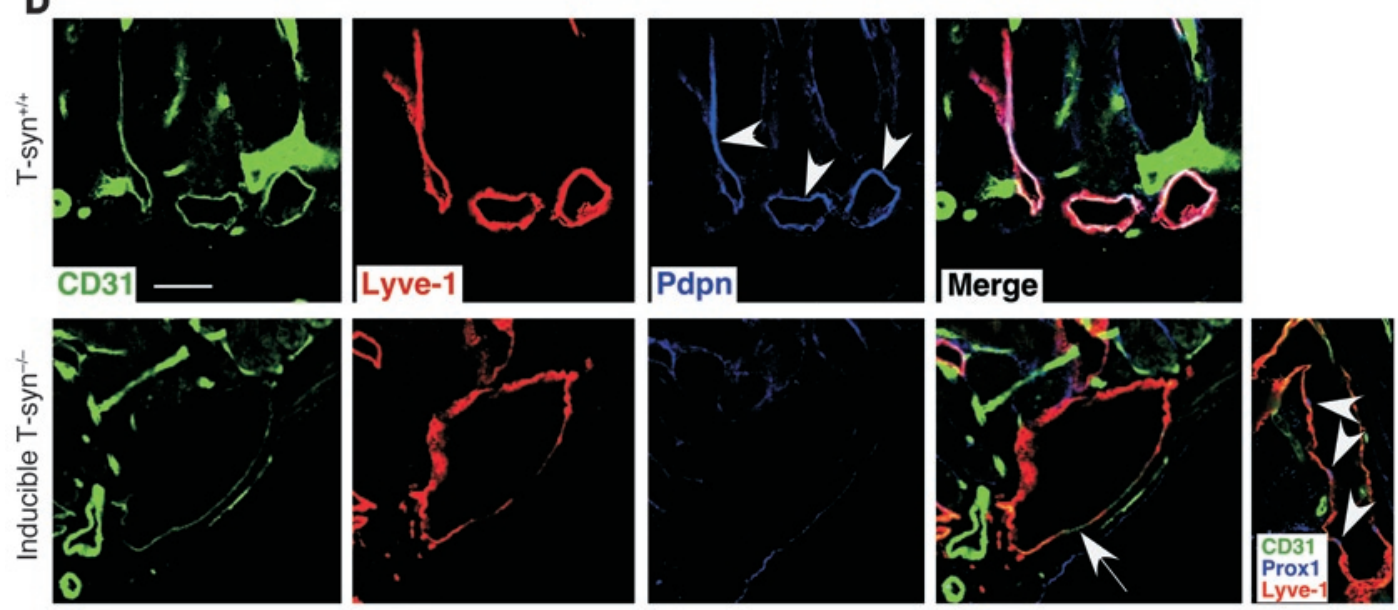

Figure 7

Postnatal abrogation of O-glycan formation results in impaired expression of podoplanin and lymphatic abnormalities that phenocopy EHC

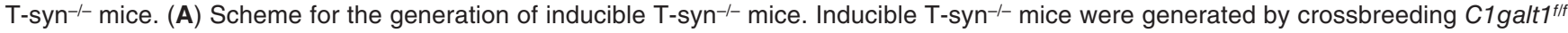
mice with $M \times 1 C r e \mathrm{Tg}$ mice in which the Cre recombinase was controlled by the $M \times 1$ promoter, which is responsive to interferon or synthetic double-strand RNA (poly-l:C). Postnatal deletion of the C1galt1 gene was achieved by injections of poly-l:C when mice were 2 weeks old. (B) Immunohistochemical staining of sections of small intestine from 7-month-old T-syn ${ }^{+/+}$and inducible $\mathrm{T}$-syn ${ }^{-/}$mice using antibodies against $\mathrm{Tn}$ or Lyve-1. Arrows indicate blood cells. (C) Intravital microscopic imaging of intestinal vascular networks after an arterial injection of India ink (8-month-old mice). (D) Confocal imaging of cryosections of small intestine from 8-month-old T-syn $+/+$ and inducible T-syn ${ }^{-/}$mice. Arrowheads mark the lymphatic endothelial podoplanin. The arrow in the merged image indicates a dilated hybrid vessel. The additional image in the inducible $\mathrm{T}^{-\mathrm{syn}^{-/}}$panel is a cryosection of intestine labeled with anti-CD31, Prox1, and Lyve-1. Arrowheads in this image indicate Prox1 staining. Data are representative of at least 3 experiments. Scale bars: $50 \mu \mathrm{m}$ (B and D); $0.5 \mathrm{~mm}$ (C).

shown). Inducible $\mathrm{T}$-syn ${ }^{-/}$lymphatic vessels had no expression of podoplanin, and the loss of lymphatic endothelial podoplanin occurred before abnormal lymphatic development (Figure 7D and data not shown), suggesting a causal relationship during postnatal tissue remodeling. These data demonstrate that O-glycosylated podoplanin is important for maintaining the separation of these systems in postnatal physiology. Therefore, abnormal O-glycosylation of podoplanin may contribute to lymphatic abnormalities in pathological conditions.

Abnormal connections of portal venous and intestinal lymphatic systems cause aberrant transport of lipids with consequent fatty liver disease. Separation of blood and lymphatic vascular systems is essential for proper transport of lipids. At P7, T-syn ${ }^{+/+}$mesenteric collecting lymphatics were filled with lymph; in contrast, EHC T-syn ${ }^{-/-}$mesenteric collecting lymphatics were filled with blood (Figure 8A). We thus examined whether these abnormalities caused abnormal lipid transport in EHC T-syn ${ }^{-/}$mice. Similar to T-syn ${ }^{+/+}$livers,
E18.5 and P0 EHC T-syn-/- livers had normal morphology (Figure $8, \mathrm{~B}$ and $\mathrm{C}$ ). However, immediately after pups began nursing on milk, the P1 EHC T-syn ${ }^{-/-}$livers displayed abnormal accumulation of vacuoles of triglycerides (Figure 8C), which resembles microvesicular steatosis in human steatosis hepatitis (34). The phenotype worsened progressively. At P7, EHC T-syn-/- livers had extensive steatosis, inflammatory infiltrates, and hepatocyte ballooning (Figure 8, B and D, and Supplemental Figure 10A). EHC $\mathrm{T}_{-} \mathrm{syn}^{-/-}$mice that survived beyond neonatal development developed liver cirrhosis and splenomegaly (Supplemental Figure 10, $\mathrm{B}$ and $\mathrm{C}$, and data not shown). EHC T-syn ${ }^{-/-}$adult mice were not obese (Supplemental Figure 10, D and E), differing from mice with Prox1 haploinsufficiency (35). The plasma lipid levels of T-syn ${ }^{+/+}$ and $\mathrm{EHC} \mathrm{T-syn}{ }^{-/}$mice were comparable (Figure 8E), suggesting no major differences in lipid metabolism. But triglyceride levels were significantly elevated in EHC T-syn ${ }^{-/}$compared with $\mathrm{T}$-syn ${ }^{+/+}$liver (Figure 8E). Given that triglycerides are the major components of 
A

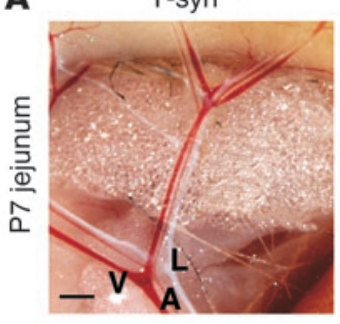

C

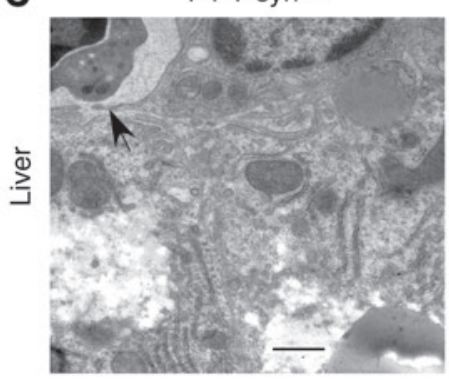

EHC T-syn-1-

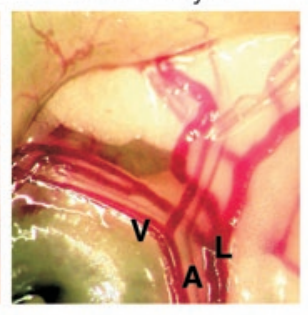

B

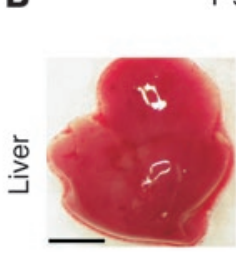

PO
T-syn+t+

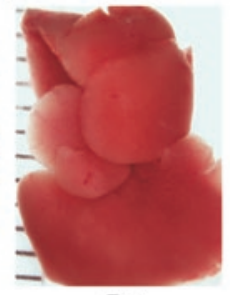

P7

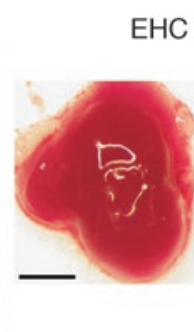

Po

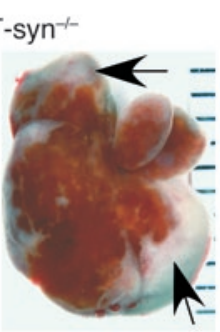

P7

P1 EHC T-syn ${ }^{-1}$
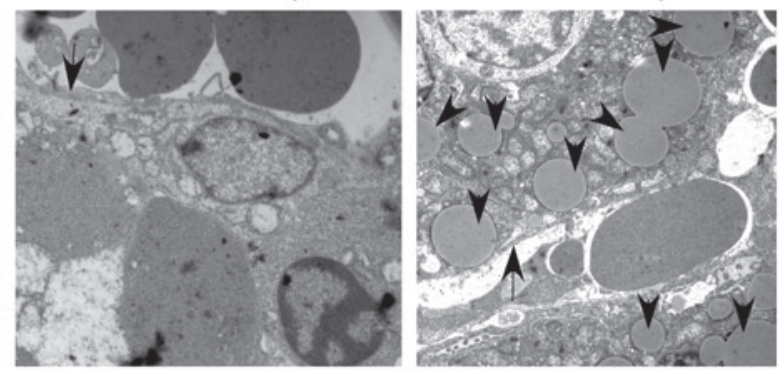

D

T-syn ${ }^{+/+}$

EHC T-syn ${ }^{-1}$
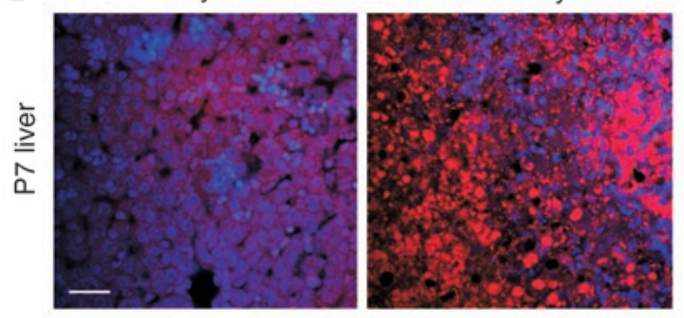

E

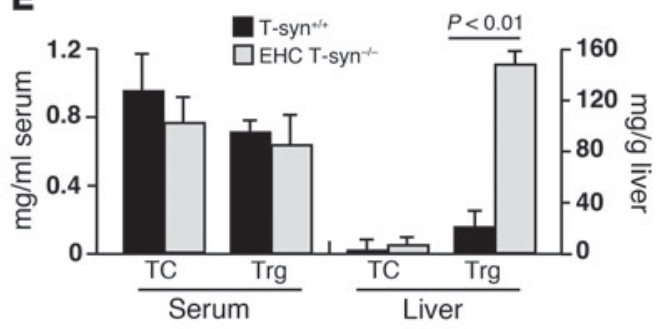

$\mathbf{F}$
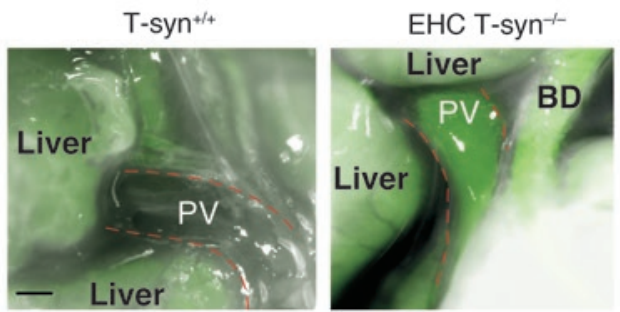

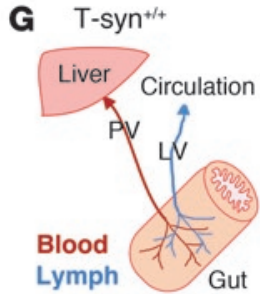

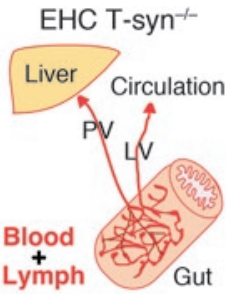

\section{Figure 8}

EHC T-syn ${ }^{-1-}$ mice develop fatty liver disease. (A) Representative images of mesenteric vessels. (B) EHC T-syn ${ }^{-/}$mice develop fatty livers. Arrows indicate white-colored areas of lipid deposition in the P7 EHC T-syn ${ }^{-/}$liver. (C) Representative transmission electron microscopic images of T-syn ${ }^{+/+}$and EHC T-syn ${ }^{-/-}$livers. Arrows indicate liver sinusoidal endothelium. Arrowheads mark lipid inclusions. (D) Abnormal lipid deposition in liver of EHC T-syn ${ }^{-1-}$ mice revealed by Nile red staining (red). (E) Lipid analysis of serum and liver tissues. TC, total cholesterol; Trg, triglycerides. (F) The portal vein (PV) of a 7-week-old EHC T-syn ${ }^{-1-}$ mouse, but not of a T-syn ${ }^{+/+}$mouse, was visualized after mice were gavaged with the fluorescent lipid BODIPY FL $\mathrm{C}_{16}$. BD, bile duct. (G) Model illustrating how misconnections of intestinal blood and lymphatic vessels leads to abnormal lipid transport in EHC T-syn ${ }^{-/}$mice. Data represent the mean \pm SEM of 3 independent experiments $(n=3-5$ mice per group). Scale bars: $2 \mathrm{~mm}$ (A and $\mathbf{B}) ; 2 \mu \mathrm{m}(\mathbf{C}) ; 50 \mu \mathrm{m}(\mathbf{D}) ; 1 \mathrm{~mm}(\mathbf{F})$.

chylomicrons, these data suggested that misconnections between intestinal blood and lymphatic vessels in EHC T-syn ${ }^{-/}$mice allowed a portion of chylomicrons to be directly transported from small intestine into the liver through the portal vein system. To test this possibility, both T-syn ${ }^{+/+}$and EHC T-syn ${ }^{-/}$mice were gavaged with the fluorescent lipid BODIPY FL $\mathrm{C}_{16}$. One hour later they were examined for the presence of fluorescent lipid in the portal vein, which normally should not be observed. As expected, the fluorescent lipids were only visualized in EHC T-syn ${ }^{-/-}$portal vein (Figure 8 F), providing direct evidence that some chylomicrons are directly deposited into liver because of misconnections between intestinal blood and lymphatic vessels (Figure 8G). Inducible T-syn ${ }^{-/-}$livers also had an increase in lipid deposition (data not shown), which is consistent with the fatty liver phenotype in EHC T-syn ${ }^{-/}$mice.

\section{Discussion}

Our results demonstrate that endothelial O-glycans control the separation of blood and lymphatic vessels during embryonic and postnatal development, at least in part by regulating podoplanin expression. Moreover, we show that intestinal blood and lymphatic 
misconnections result in abnormal lipid transport and consequent fatty liver disease in $\mathrm{EHC} \mathrm{T-syn}{ }^{-/-}$mice.

Although widely present in most cell types/tissues, O-glycans are highly expressed in endothelial and hematopoietic cells throughout embryonic and postnatal development $(19,20,22)$. $\mathrm{T}^{-} \mathrm{syn}^{-/-}$embryos die around E12.5 because of massive bleeding and defective angiogenesis in neural tissues (22). Unlike T-syn ${ }^{-/}$ mice, EHC T-syn ${ }^{-/-}$mice do not have brain bleeding and have partial late-onset embryonic lethality. This phenotypic difference may be attributable to O-glycans in other cell types such as mural cells and neural cells that may also contribute to vascular development in neural tissues. In addition, other factors such as variations in genetic backgrounds and the temporal difference in C1galt1 inactivation between global T-syn ${ }^{-1-}$ mice and EHC $\mathrm{T}^{-} \mathrm{syn}^{-/}$mice (inactivation occurs around E8) might also contribute to the phenotypic difference.

EHC T-syn ${ }^{-/-}$mice have defects in lymphatic development and function, with misconnected blood and lymphatic vessels. Such blood-filled lymphatic vessels have been observed in mice with deficiencies of the signaling proteins Syk and SLP-76 and in mice lacking fasting-induced adipose factor $(15,28)$. However, these molecules are not considered to be expressed in endothelial cells $(15,36)$. Angiogenesis and lymphangiogenesis progress side by side utilizing common mechanisms including guided endothelial cell sprouting and fusion to form specific vascular networks $(2,3,14,37)$. Hence, there likely exist endothelial autonomous mechanisms to prevent fusion between these two similar but distinct endothelial cell types. Our observation of hybrid microvessels in EHC T-syn ${ }^{-1-}$ mice suggests that endothelial O-glycans are involved in such a mechanism. All the endothelial cells of these hybrid vessels expressed Prox1, which is a marker for the definitive commitment to lymphatic endothelium (11). However, a substantial number of the Prox1-positive cells lost their expression of Lyve-1, which is a reliable differentiation marker for intestinal lymphatic microvessels. The Prox1positive cells also displayed increased expression of CD31 as well as ectopic expression of blood vascular markers such as CD105 and endomucin. Taken together, these data indicate that a loss of lymphatic endothelial identity is the primary cause of the formation of the hybrid vessels in EHC T-syn ${ }^{-1-}$ mice. The observed marker profile further suggests that endothelial O-glycans are dispensable for the early commitment phase of endothelial cells to the lymphatic lineage but are indispensable for the subsequent endothelial differentiation and maturation that are required for development and maintenance of segregated blood and lymphatic vessels. Although unlikely, a potential alternative explanation could be that misconnections initially arise independent of identity loss and that the confused endothelial marker profile is a consequence of altered flow dynamics in EHC $\mathrm{T}_{-} \mathrm{syn}^{-/-}$lymphatic vessels resulting from the misconnections. Whether misconnections arise at specific sites or sporadically remains to be investigated.

The molecular mechanism of the lymphatic defects in EHC $\mathrm{T}$-syn ${ }^{-/-}$mice appears to be related to the impaired expression of podoplanin. The observed lymphatic abnormalities in EHC

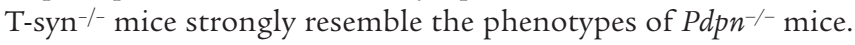
Postnatal deletion of O-glycans in inducible $\mathrm{T}$-syn ${ }^{-/}$mice also resulted in impaired expression of endothelial podoplanin that occurs prior to the onset of lymphatic defects. Thus, the loss of podoplanin is likely a cause rather than consequence of lymphatic defects. It is worth noting that the lymphatic phenotype in inducible $\mathrm{T}_{-} \mathrm{syn}^{-/}$mice is primarily observed in small intestines, where tissue remodeling and angiogenesis are active postnatally. These observations suggest that the maintenance of segregated blood and lymphatic vessels requires persistent expression of O-glycoproteins, such as podoplanin, in tissues of continuous turnover of lymphatic endothelial cells in adult mice. In addition, these findings underscore the potential pathophysiological relevance of this unique phenotype. Taken together, these results indicate that O-glycans are specifically required in lymphatic endothelial cells, primarily to ensure proper function of podoplanin for the establishment and maintenance of distinct blood and lymphatic systems during embryonic development and postnatal tissue remodeling. Although lack of O-glycosylation might affect the functions of other glycoproteins that regulate lymphangiogenesis and thus contribute to the vascular defects of EHC T-syn ${ }^{-/}$mice, our results indicate that abnormal O-glycosylation of endothelial podoplanin is sufficient to result in the formation of hybrid vessels and blood/ lymphatic vessel misconnections.

Prox 1 is expressed in both transitional and mature lymphatic endothelial cells and controls the establishment of lymphatic systems during embryonic development $(11,13,38)$. Yet it remains unclear how Prox1 controls the switch from venous endothelial cells to lymphatic endothelial cells and how it maintains lymphatic endothelial cell identity. Intriguingly, lymphatic endothelial cells that lack O-glycans have impaired expression of podoplanin and express a mosaic of blood endothelial and lymphatic endothelial markers. This result suggests that O-glycosylation of podoplanin contributes to lymphatic endothelial cell identity. The Pdpn gene is one of the target genes whose expression is upregulated by Prox 1 (39). Therefore, it is plausible that Prox 1 controls the segregation of blood and lymphatic systems, at least in part by upregulating podoplanin expression. Podoplanin induces aggregation of platelets that express Syk and SLP-76 by interacting with a lectin-like receptor named CLEC-2 on platelets (40-42). However, podoplanin likely controls additional processes that are independent of platelets, as mice having either no or less than $15 \%$ circulating platelets do not display lymphatic defects (43-45). Therefore, precisely how podoplanin regulates lymphatic development remains to be defined.

Lipid transport is one of the primary functions of lymphatic vessels $(10,11)$. Dietary lipids are transported in the form of chylomicrons from small intestine to systemic circulation via the intestinal lymphatic vessels and thoracic duct. In circulation, triglyceride components of chylomicrons are unloaded to adipose and muscle tissues by the activity of lipoprotein lipase (46). Consequently, only chylomicron remnants are transported to liver through the hepatic artery. In EHC T-syn ${ }^{-/}$mice, because of aberrant intestinal vein and lymphatic connections, chylomicrons are directly transported to liver via the portal vein system, which causes fatty liver disease (steatohepatitis) (Figure 8G). Nonalcoholic fatty liver disease is prevalent $(34,47)$. However, the pathogenesis of this disease remains poorly understood $(34,48)$. Our findings provide what we believe to be a novel potential mechanism for the pathogenesis of this common disease.

In conclusion, endothelial O-glycans control the separation of blood and lymphatic vessels during embryonic and postnatal development, at least in part by regulating podoplanin expression. Aberrant connections between intestinal blood and lymphatic vessels contribute to the pathogenesis of fatty liver disease in mice. 


\section{Methods}

Mice, microscopy, semiquantitative RT-PCR, and morpholino knockdown in Xenopus laevis and zebrafish. See Supplemental Methods.

Mice. To generate EHC T-syn ${ }^{-/-}$mice, C1galt1 $1 / f$ mice were crossed with Tie2Cre Tg mice [Tg(Tek-cre)1Ywa] (provided by Masashi Yanagisawa, University of Texas Southwestern Medical Center, Dallas, Texas, USA) $(23,24)$. Since the Tie2Cre transgene is expressed in the female germline (49), C1galt1 ${ }^{\mathrm{f} /}{ }^{+} \mathrm{Tie} \mathrm{Cr} \mathrm{C}^{+}$males and C1galt $1 \mathrm{f} / \mathrm{f}$ females were used to generate EHC T-syn ${ }^{-/-}$mice. Inducible T-syn ${ }^{-/-}$mice were generated by crossing C1galt1f/f mice with Mx1Cre Tg mice [Tg(Mx1-cre)1Cgn; The Jackson Laboratory] (50). To induce the deletion of C1galt1 in vivo, mice were injected intraperitoneally with $250 \mu \mathrm{g}$ of poly-I:C (Sigma-Aldrich) once every other day over 6 days ( 3 injections total) starting at age 2 weeks. $P d p n^{-/-}$mice (129/SvEv background) were generated as described previously (33) and provided by M.C. Williams (Boston University School of Medicine, Boston, Massachusetts, USA).

Endothelial cell-specific C1galt1 Tg mice (Tie2-T-syn Tg) on an FVB/N genetic background were generated with standard procedures. In brief, full-length murine C1galt1 was inserted into the vector that contained the 2.1-kb promoter and 10-kb enhancer of the gene Tie2 (a gift from Thomas Sato, Weill Medical College of Cornell University, New York, New York, USA) (30). The purified DNA fragment was microinjected into a fertilized FVB/N mouse oocytes, and offspring were produced. Mice were genotyped by PCR using the following primers: a (5'-ATGGCCTCTAAATCTTGGCTG-3') and b (5'-TCATGTATCCCTGCTTCAC-3'), which effectively amplify the mouse C1galt1 cDNA but not genomic DNA. To generate rescued EHC T-syn ${ }^{-/-}$mice, Tie2-T-syn Tg mice were crossed with EHC T-syn ${ }^{+/-}$mice. Primers c (5'-TGACAGCCAGGAATGGAACTTG-3') and d (5'-GCCTCTTCTCGCAACAAAATACTC-3') were used to detect floxed exon 1 of the C1galt1 gene.

Mice were kept in a specific pathogen-free barrier facility. Genotypes of mice were determined by PCR of DNA from tail biopsies. Unless specified, all mice were of mixed genetic background (129S1/SvIm and C57BL/6J). Sex-matched WT littermate controls (C1galt1f//Tie2Cre ${ }^{-}$or C1galt1f/+ Tie2Cre ; all are referred to as $\mathrm{T}-\mathrm{syn}^{+/+}$) were used for all experiments. Animal studies were approved by the IACUCs of the Oklahoma Medical Research Foundation and the Katholieke Universiteit Leuven.

Endothelial cells. Primary endothelial cells were isolated from lung and skin as described previously, with minor modifications (51). Briefly, the finely minced lung or skin tissue was digested with enzyme solution $(2 \mathrm{mg} / \mathrm{ml}$ collagenase, $5 \mathrm{mg} / \mathrm{ml}$ dispase; Roche Applied Science) at $37^{\circ} \mathrm{C}$ for 1 hour, and the cells were cultured in complete endothelial cell medium (Invitrogen). Endothelial cells were purified from culture with Dynabeads M-450 (Dynal, Invitrogen) that were coated with rat anti-mouse CD102 (clone 3C4; BD) according to the manufacturer's instructions. Subconfluent primary endothelial cells were used for experiments at passages 1 or 2 . Endothelial cell purity (>95\%) was measured by expression of CD31 and CD102.

To establish endothelial cell lines, endothelial cells were transfected with plasmid pPSVE1-B1a (from Jeffrey D. Esko, UCSD, La Jolla, California, USA), carrying SV40 viral T antigen using Lipofectin (Invitrogen). The cells at passages 5-10 were used for experiments.

$T$-synthase activity and $O$-glycan structure. T-synthase enzymatic activity was measured using GalNAco1-O-phenyl (Sigma-Aldrich) as an acceptor, as described previously $(19,22)$.

O-glycan structures from cultured endothelial cells were analyzed as described previously (52). Briefly, T-syn ${ }^{+/+}$and T-syn ${ }^{-/-}$endothelial cell lines were collected and vacuum dried, and O-glycans were released by ammonia-based $\beta$-elimination. The glycans were labeled with 2 -aminobenzamide (2-AB) and collected from phase columns (Zorbax NH2; Agilent Technologies) using HPLC equipped with a fluorescence detector (excitation,
$330 \mathrm{~nm}$; emission, $420 \mathrm{~nm}$ ). Glycans were analyzed for composition/structure by MALDI-TOF-MS (Applied Biosystems) in a linear positive mode.

Microscopy. Embryos and organs were photographed at autopsy. For routine histology, tissues were fixed with $10 \%$ formalin and embedded in paraffin. Sections $(5 \mu \mathrm{m})$ were stained with H\&E.

For immunohistochemistry, deparaffinized sections were blocked with a serum-free protein block (Dako) and streptavidin/biotin blocking kit (Vector Laboratories). Sections were then incubated overnight with biotinylated goat anti-mouse Lyve-1 (5 $\mu \mathrm{g} / \mathrm{ml}$; R\&D Systems) or biotinylated anti-Tn antigen $\mathrm{mAb}(2 \mu \mathrm{g} / \mathrm{ml}$; clone Ca3638, IgM) as the primary antibody overnight at $4{ }^{\circ} \mathrm{C}$. The staining was visualized using HRP-conjugated streptavidin (Dako) and diaminobenzidine substrate (Vector Laboratories).

For confocal microscopy, mouse tissues were fixed in 4\% PFA at room temperature for 1-2 hours, washed, cryoprotected with $15 \%$ sucrose at $4{ }^{\circ} \mathrm{C}$ overnight, and mounted in OCT compound (Sakura Finetek). Cryosections (approximately 20-50 $\mu \mathrm{m}$ ) were incubated with biotin-conjugated goat anti-mouse Lyve-1 $(5 \mu \mathrm{g} / \mathrm{ml})$, rat anti-mouse CD31 mAb (clone MEC13.3, $1: 50$; BD), and Cy3-conjugated anti- $\alpha$-SMA mAb (clone 1A4; 1:50; SigmaAldrich) overnight at $4^{\circ} \mathrm{C}$; developed with streptavidin Cy5 (1:50; Invitrogen) and Alexa Fluor 488-conjugated donkey anti-rat IgG (1:100; Invitrogen); and mounted with ProLong Gold mounting medium supplemented with ToPro3 (Invitrogen). Sections were also stained with biotin-conjugated goat anti-mouse Lyve-1, rat anti-mouse CD31 mAb, hamster anti-podoplanin $\mathrm{mAb}$ (clone 8.1.1; 4 $\mu \mathrm{g} / \mathrm{ml}$; Santa Cruz Biotechnology Inc.), rat anti-mouse CD105 mAb (clone MJ7/18; $5 \mu \mathrm{g} / \mathrm{ml}$; eBioscience), rat anti-mouse endomucin $\mathrm{mAb}$ (clone V7C7; $2 \mu \mathrm{g} / \mathrm{ml}$; Santa Cruz Biotechnology Inc.), and rabbit anti-Prox 1 (1:100; Abcam) in various combinations. For whole-mount staining, small intestines were fixed overnight in 2\% PFA, and the tissues were treated with $3 \%$ BSA in serum-free protein block with $0.3 \%(v / v)$ Triton $\mathrm{X}-100$ and subsequently incubated with rat anti-mouse CD31 mAb, hamster anti-mouse podoplanin mAb, biotinylated goat anti-mouse Lyve-1, or rabbit anti-Prox 1 . The samples were analyzed by confocal laser scanning microscopy using a Nikon C1 scanning head mounted on a Nikon ECLIPSE $2000 \mathrm{U}$ inverted microscope (Plan Apochromats dry objective lens, $\times 20$, NA 0.75; Nikon Instruments Inc.). Z-stack images were collected at $1-\mu \mathrm{m}$ steps with sequential laser excitation to eliminate bleed-through and with confocal parameters selected to minimize the thickness of the calculated optical section. Volume images from the confocal data sets were processed with IMARIS software (Bitplane) for 3-dimensional views of detailed vascular morphology. Images are presented as maximum-intensity projections of the Z-stacks. Hybrid vessels in EHC T-syn ${ }^{-/-}$mice were identified based on their mixed expression of blood and lymphatic markers. Confocal images at $\times 20$ were used for quantification.

For transmission electron microscopy, specimens were fixed with $2 \%$ glutaraldehyde and 3\% PFA solution, postfixed for 90 minutes with 1\% osmium tetroxide, and then treated with $1 \%$ tannic acid (all in $0.1 \mathrm{M}$ sodium cacodylate- $\mathrm{HCl}$ buffer, $\mathrm{pH}$ 7.2). After dehydration in graded ethanol and propylene oxide treatment, specimens were embedded in Epon 812 (TAAB), sectioned with an ultramicrotome, and examined with an H-7600 transmission electron microscope (Hitachi).

For intravital microscopy, mice were anesthetized with an intraperitoneal injection of tribromoethanol and amylene hydrate (SigmaAldrich) and placed on a heating pad $\left(37^{\circ} \mathrm{C}\right)$ to maintain body temperature. A segment of small intestine was exteriorized, and $200 \mu \mathrm{l}$ of lysine fixable dextran-FITC (150 kDa at $1 \mathrm{mg} / \mathrm{ml}$; Invitrogen), India ink, or red fluorescent microbeads (diameter, $1 \mu \mathrm{m}$; Invitrogen) were injected via the left common carotid artery catheter. Intravital microscopy was conducted using an Eclipse E600FN microscope (Nikon) with a saline immersion $\times 4$ objective. The microscope was equipped with a DC-330 CCD color camera (Dage-MTI) connected to a video recorder (Panasonic). Video was 
recorded for subsequent frame-by-frame analysis to capture the arterial and venous phases of the flow.

Immunoblotting. Primary endothelial cells from lungs were lysed with RIPA buffer (Pierce) supplemented with complete proteinase inhibitor cocktail (Roche Applied Science). Protein concentration was quantified using the micro BCA protein assay kit (Pierce), and cell lysates containing $25 \mu \mathrm{g}$ total protein were resolved by $5 \%-20 \%$ gradient SDS-PAGE. After blotting, Immobilon-P transfer membranes (Millipore) were blocked and probed with the following antibodies: anti-podoplanin, anti-Tie1, antiTie2, anti-Ang2, anti-VEGF-C (Santa Cruz Biotechnology Inc.), anti-Lyve-1 (Novus Biologicals), anti-VEGFR3 (Abcam), anti-Prox1 (Abgent), anti-integrin $\alpha 9$ (R\&D Systems), anti-FIAF (Zymed, Invitrogen), and anti- $\beta$-actin (US Biologicals). For lectin blotting, cell lysates were immunoprecipitated with anti-podoplanin. The immunoprecipitated podoplanin was subjected to SDS-PAGE and transferred to a membrane and then probed with biotinconjugated lectin Helix pomatia agglutinin (10 $\mu \mathrm{g} / \mathrm{ml}$; EY Laboratories Inc.). Signal was developed with ECL detection reagents (Amersham).

Lipid analysis. After a 4-hour fast, mouse blood was obtained by retroorbital bleeding. Serum total cholesterol and triglyceride levels were measured by Infinity cholesterol reagent and Infinity triglyceride reagent, respectively (Thermo Scientific). Lipids in liver were analyzed as described previously (35). Briefly, liver tissue ( $100 \mathrm{mg}$ ) was collected from P7 pups, homogenized, and extracted with chloroform/methanol. After resuspension of the lipid in distilled water, the levels of cholesterol and triglycerides were determined as described previously.

For lipid staining, cryosections (10 $\mu$ m thick) of liver were used for Nile red (Invitrogen) staining to reveal lipid droplets as described previously (53). For analysis of lipid transportation, mice were gavaged with BODIPY $\mathrm{FL} \mathrm{C}_{16}$ (40 $\mu \mathrm{g}$ in $200 \mu \mathrm{l}$ solution; Invitrogen) (35). After 1 hour, mice were anesthetized and the transport of fluorescent lipid was visualized under intravital microscopy.

Statistics. Results are presented as mean \pm SEM. Two-tailed Student's $t$ test was used to assess potential differences between 2 groups. We considered $P<0.05$ to be significant.

\section{Acknowledgments}

We thank Rodger McEver and Charles Esmon for insightful suggestions and critical reading of the manuscript. We thank Susannah Rankin for her help with Xenopus database analysis and Tao Wen and Todd Walker for technical support. We thank Jeffrey Esko for reagents and Mary C. Williams for providing $P d p n^{-/-}$mice. Microinjection of oocytes was performed at the Oklahoma Medical Research Foundation microinjection facility, directed by Ute Hochgeschwender. Tissue processing and electron microscopy were performed in the imaging core facility of the Oklahoma Medical Research Foundation. This work was supported by NIH grants HL085607 to L. Xia; RR018758 to L. Xia, R.D. Cummings, and F. Lupu; and R01 GM37704 to F. Lupu. H. Gerhardt and E. Nye are supported by Cancer Research UK. H. Gerhardt is supported by the EMBO Young Investigator Program.

Received for publication April 30, 2008, and accepted in revised form September 10, 2008.

Address correspondence to: Lijun Xia, Cardiovascular Biology Research Program, Oklahoma Medical Research Foundation, 825 NE 13th Street, Oklahoma City, Oklahoma 73104, USA. Phone: (405) 271-7892; Fax: (405) 271-3137; E-mail: lijun-xia@omrf.org.
1. Jain, R.K. 2003. Molecular regulation of vessel maturation. Nat. Med. 9:685-693.

2. Carmeliet, P. 2005. Angiogenesis in life, disease and medicine. Nature. 438:932-936.

3. Gerhardt, H., and Betsholtz, C. 2005. How do endothelial cells orientate? In Mechanisms of angiogenesis. M. Clauss and G. Breier, editors. Birkhäuser Verlag. Basel, Switzerland. 3-15.

4. Sato, T.N. 2003. Vascular development: molecular logic for defining arteries and veins. Curr. Opin. Hematol. 10:131-135.

5. Hynes, R.O. 2002. A reevaluation of integrins as regulators of angiogenesis. Nat. Med. 8:918-921.

6. Yancopoulos, G.D., et al. 2000. Vascular-specific growth factors and blood vessel formation. Nature. 407:242-248.

7. Coultas, L., Chawengsaksophak, K., and Rossant, J. 2005. Endothelial cells and VEGF in vascular development. Nature. 438:937-945.

8. You, L.R., et al. 2005. Suppression of Notch signalling by the COUP-TFII transcription factor regulates vein identity. Nature. 435:98-104.

9. Hofmann, J.J., and Iruela-Arispe, M.L. 2007. Notch signaling in blood vessels: who is talking to whom about what? Circ. Res. 100:1556-1568.

10. Alitalo, K., Tammela, T., and Petrova, T.V. 2005. Lymphangiogenesis in development and human disease. Nature. 438:946-953.

11. Oliver, G., and Alitalo, K. 2005. The lymphatic vasculature: recent progress and paradigms. Annu. Rev. Cell Dev. Biol. 21:457-483.

12. Alitalo, K., and Carmeliet, P. 2002. Molecular mechanisms of lymphangiogenesis in health and disease. Cancer Cell. 1:219-227.

13. Wigle, J.T., and Oliver, G. 1999. Prox1 function is required for the development of the murine lymphatic system. Cell. 98:769-778.

14. Iruela-Arispe, M.L. 2006. When it comes to blocking lymphatics, it is all a question of time. Am. J.
Pathol. 169:347-350.

15. Abtahian, F., et al. 2003. Regulation of blood and lymphatic vascular separation by signaling proteins SLP-76 and Syk. Science. 299:247-251.

16. Detmar, M., and Hirakawa, S. 2002. The formation of lymphatic vessels and its importance in the setting of malignancy. J. Exp. Med. 196:713-718.

17. Bischoff, J. 1997. Cell adhesion and angiogenesis. J. Clin. Invest. 100:S37-S39.

18. Julenius, K., Molgaard, A., Gupta, R., and Brunak, S. 2005. Prediction, conservation analysis, and structural characterization of mammalian mucin-type O-glycosylation sites. Glycobiology. 15:153-164.

19. Ju, T., Brewer, K., D’Souza, A., Cummings, R.D. and Canfield, W.M. 2002. Cloning and expression of human core $1 \beta 1,3$-galactosyltransferase. J. Biol. Chem. 277:178-186.

20. Ju, T., and Cummings, R.D. 2002. A unique molecular chaperone Cosmc required for activity of the mammalian core 1 beta 3-galactosyltransferase. Proc. Natl. Acad. Sci. U. S. A. 99:16613-16618.

21. Varki, A., et al. 1999. Essentials of glycobiology. Cold Spring Harbor Laboratory Press. Cold Spring Harbor, New York, USA. 653 pp.

22. Xia, L., et al. 2004. Defective angiogenesis and fatal embryonic hemorrhage in mice lacking core 1 derived O-glycans. J. Cell Biol. 164:451-459.

23. Williams, S.A., Xia, L., Cummings, R.D., McEver, R.P., and Stanley, P. 2007. Fertilization in mouse does not require terminal galactose or $\mathrm{N}$-acetylglucosamine on the zona pellucida glycans. J. Cell Sci. 120:1341-1349.

24. Kisanuki, Y.Y., et al. 2001. Tie2-Cre transgenic mice: a new model for endothelial cell-lineage analysis in vivo. Dev. Biol. 230:230-242.

25. Baluk, P., and McDonald, D.M. 2008. Markers for microscopic imaging of lymphangiogenesis and angiogenesis. Ann. N. Y. Acad. Sci. 1131:1-12.

26. Jackson, D.G., Prevo, R., Clasper, S., and Banerji,
S. 2001. LYVE-1, the lymphatic system and tumor lymphangiogenesis. Trends Immunol. 22:317-321.

27. Makinen, T., et al. 2005. PDZ interaction site in ephrinB2 is required for the remodeling of lymphatic vasculature. Genes Dev. 19:397-410.

28. Backhed, F., Crawford, P.A., O'Donnell, D., and Gordon, J.I. 2007. Postnatal lymphatic partitioning from the blood vasculature in the small intestine requires fasting-induced adipose factor. Proc. Natl. Acad. Sci. U. S. A. 104:606-611.

29. Jones, C.A., et al. 2008. Robo4 stabilizes the vascular network by inhibiting pathologic angiogenesis and endothelial hyperpermeability. Nat. Med. 14:448-453.

30. Schlaeger, T.M., et al. 1997. Uniform vascularendothelial-cell-specific gene expression in both embryonic and adult transgenic mice. Proc. Natl. Acad. Sci. U. S. A. 94:3058-3063.

31. Motoike, T., et al. 2000. Universal GFP reporter for the study of vascular development. Genesis. 28:75-81.

32. Schacht, V., et al. 2003. T1 $\alpha /$ podoplanin deficiency disrupts normal lymphatic vasculature formation and causes lymphedema. EMBO J. 22:3546-3556.

33. Ramirez, M.I., et al. 2003. T1 $\alpha$, a lung type I cell differentiation gene, is required for normal lung cell proliferation and alveolus formation at birth. Dev. Biol. 256:61-72.

34. Angulo, P. 2002. Nonalcoholic fatty liver disease. N. Engl. J. Med. 346:1221-1231.

35. Harvey, N.L., et al. 2005. Lymphatic vascular defects promoted by Prox 1 haploinsufficiency cause adultonset obesity. Nat. Genet. 37:1072-1081.

36. Sebzda, E., et al. 2006. Syk and Slp-76 mutant mice reveal a cell-autonomous hematopoietic cell contribution to vascular development. Dev. Cell. 11:349-361.

37. Adams, R.H., and Alitalo, K. 2007. Molecular regulation of angiogenesis and lymphangiogenesis. Nat. 
Rev. Mol. Cell Biol. 8:464-478.

38. Wigle, J.T., et al. 2002. An essential role for Prox1 in the induction of the lymphatic endothelial cell phenotype. EMBO J. 21:1505-1513.

39. Hong, Y.K., et al. 2002. Prox1 is a master control gene in the program specifying lymphatic endothelial cell fate. Dev. Dyn. 225:351-357.

40. Kaneko, M., et al. 2004. Functional sialylated O-glycan to platelet aggregation on Aggrus (T1alpha/Podoplanin) molecules expressed in Chinese hamster ovary cells. J. Biol. Chem. 279:38838-38843.

41. Suzuki-Inoue, K., et al. 2006. A novel Syk-dependent mechanism of platelet activation by the C-type lectin receptor CLEC-2. Blood. 107:542-549.

42. Suzuki-Inoue, K., et al. 2007. Involvement of the snake toxin receptor CLEC-2, in podoplanin-mediated platelet activation, by cancer cells. J. Biol. Chem. 282:25993-26001.

43. Shivdasani, R.A., et al. 1995. Transcription factor
NF-E2 is required for platelet formation independent of the actions of thrombopoietin/MGDF in megakaryocyte development. Cell. 81:695-704.

44. Gurney, A.L., Carver-Moore, K., de Sauvage, F.J., and Moore, M.W. 1994. Thrombocytopenia in c-mpl-deficient mice. Science. 265:1445-1447.

45. de Sauvage, F.J., et al. 1996. Physiological regulation of early and late stages of megakaryocytopoiesis by thrombopoietin. J. Exp. Med. 183:651-656.

46. Black, D.D. 2007. Development and physiological regulation of intestinal lipid absorption. I. Development of intestinal lipid absorption: cellular events in chylomicron assembly and secretion. Am. J. Physiol. Gastrointest. Liver Physiol. 293:G519-G524.

47. Perlemuter, G., Bigorgne, A., Cassard-Doulcier, A.M., and Naveau, S. 2007. Nonalcoholic fatty liver disease: from pathogenesis to patient care. Nat. Clin. Pract. Endocrinol. Metab. 3:458-469.

48. Browning, J.D., and Horton, J.D. 2004. Molecular mediators of hepatic steatosis and liver injury. J. Clin. Invest. 114:147-152.

49. Koni, P.A., et al. 2001. Conditional vascular cell adhesion molecule 1 deletion in mice: impaired lymphocyte migration to bone marrow. J. Exp. Med. 193:741-754.

50. Kuhn, R., Schwenk, F., Aguet, M., and Rajewsky, K. 1995. Inducible gene targeting in mice. Science. 269:1427-1429.

51. Lim, Y.C., et al. 2003. Heterogeneity of endothelial cells from different organ sites in T-cell subset recruitment. Am. J. Pathol. 162:1591-1601.

52. Xia, B., Royall, J.A., Damera, G., Sachdev, G.P., and Cummings, R.D. 2005. Altered O-glycosylation and sulfation of airway mucins associated with cystic fibrosis. Glycobiology. 15:747-775.

53. Greenspan, P., Mayer, E.P., and Fowler, S.D. 1985. Nile red: a selective fluorescent stain for intracellular lipid droplets. J. Cell Biol. 100:965-973. 